\title{
A MODE-DEPENDENT ENERGY-BASED DAMAGE MODEL FOR PERIDYNAMICS AND ITS IMPLEMENTATION
}

\author{
CHRISTIAN WILLBERG, LASSE WIEDEMANN, AND MARTIN RÄDEL
}

\begin{abstract}
The mathematical modeling of failure mechanisms in solid materials and structures is a long standing problem. In recent years, Peridynamics has been used as a theoretical basis for numerical studies of fracture initiation, evolution and propagation. In order to investigate damage phenomena numerically, suitable material and damage models have to be implemented in an efficient numerical framework. This framework should be highly parallelizable in order to cope with the computational effort due to the high spatial and, depending on the problem, temporal resolution required for high accuracy. The open-source peridynamic framework Peridigm offers a computational platform upon which new developments of the peridynamic theory can be implemented. Today, isotropic material models and a very simple damage model are implemented in Peridigm.

This paper proposes three energy-based damage criteria. The implementation approach as well as the extension of Peridigm with these physically motivated models is described. The original criterion of Foster et al. [1] is adapted for ordinary state based material. The other two criteria utilize the decomposition of peridynamic states in isotropic and deviatoric parts to account for the failure-mode dependency.

The original criterion is verified by the numerical simulation of two mechanical problems. At first, a virtual double cantilever beam (DCB) experiment is performed to determine the energy release rate. This value is the fundamental material property required for the proposed criteria. Additionally, the DCB problem is then used to investigate the convergence of the numerical scheme implemented in Peridigm. In a second step, a model of a plate with a cylindrical hole under tensile loading is compared with an extended finite element method (XFEM) solution. Results of both numerical solutions are in good agreement. Finally, a fiber reinforced micro structure model is used to analyze the effect of the different criteria to the damage initiation and crack propagation under a more complex loading condition.
\end{abstract}

\section{NOMENCLATURE}

$\begin{array}{cl}m_{V} & \text { Weighted volume } \\ s & \text { Stretch } \\ s_{C} & \text { Critical stretch } \\ t & \text { Time } \\ u & \text { Displacement } \\ w & \text { Bond energy micro potential } \\ E & \text { Young's modulus } \\ G & \text { Shear modulus } \\ G_{0} & \text { Energy release rate } \\ G_{0 C} & \text { Critical energy release rate } \\ G_{I C} & \text { Critical energy release rate mode I } \\ G_{I I C} & \text { Critical energy release rate mode II }\end{array}$
$\mathcal{H}$ Family
$K$ Bulk modulus
$\mathbb{R}$ Euclidean space
$V$ Volume
$W$ Strain energy density
$\chi$ Scalar damage function
$\delta$ Horizon
$\varepsilon$ Strain
$\epsilon$ Strain tensor component
$\nu$ Poisson ratio
$\rho$ Density
$\theta$ Dilatation

Key words and phrases. Peridynamics, damage model, open source. 
$\xi$ Component of the undeformed vector state

$\boldsymbol{\eta}$ Bond vector, deformed

$\boldsymbol{\xi}$ Bond vector, undeformed

b External body force density

$\boldsymbol{\sigma}$ Cauchy stress

u Deformation vector

$\ddot{\mathbf{u}}$ Acceleration vector

$\mathbf{x}$ Position vector, undeformed

y Position vector, deformed
F Deformation gradient

$\underline{\omega}$ Influence scalar state

$\underline{e}$ Extension scalar state

$t$ Force density scalar state

$x$ Position scalar state, undeformed

y Position scalar state, deformed

$\overline{\mathbf{T}}$ Force vector state

$\underline{\mathrm{X}}$ Reference vector state

$\underline{\mathbf{Y}}$ Deformation vector state

\section{Introduction}

Today, the full exploitation of the lightweight potential of fiber reinforced plastics (FRPs) is limited due to missing reliability of failure predictions of real structures. Compared to isotropic materials, the failure mechanisms in FRPs are very complex, as shown in Figure 1a and Figure 1b. Figure 1a shows the crack pattern of an uni-axial test of FRPs specimen including fiber ondulations as typical manufacturing deviations. The crack is curved and multiple delaminations occur if the specimen fails. Figure 1b illustrates a FRPs microstructure under tensile loading perpendicular to the fiber direction. In such specimens the crack starts at multiple locations with the largest stress concentrations [2]. If the load is increased further, the local cracks begin to merge, resulting in the complete failure of the specimen [3].

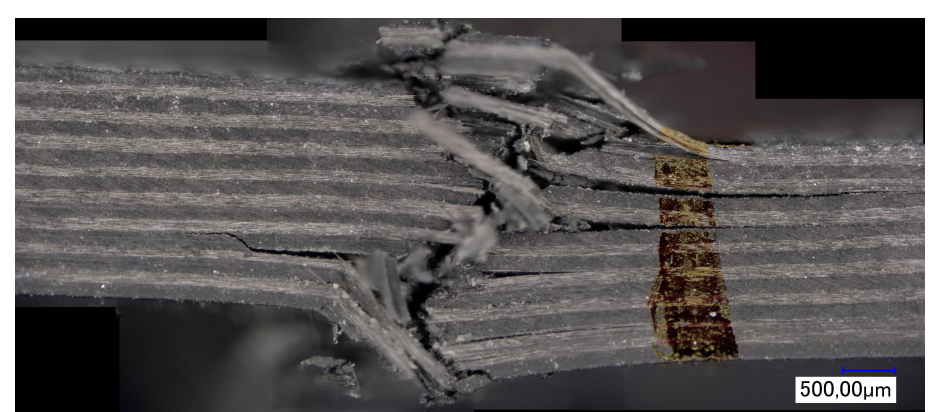

A) Crack in a CFRP specimen. Courtesy of DLR.

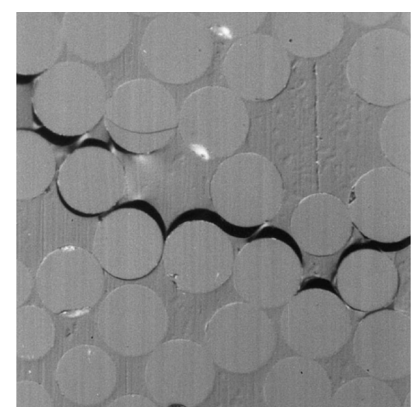

B) Matrix failure $[2,3]$

FIgURE 1. Exemplary failure mechanisms in FRP materials

Accurate modeling of such damage and fracture phenomena, including static and dynamic crack propagation, is an active and open challenge among researchers. The main difficulty inherent in such problems arise from the fact that crack nucleation and propagation in materials cannot be accurately predicted by the majority of currently available computational techniques based on classical continuum theory of mechanics [4].

The current state-of-the-art methods used in industry and research for failure prediction are based on classical continuum mechanics $(\mathrm{CM})$ and its numerical implementation in the finite element method (FEM). The classical CM is well suited for stress analyzes of undamaged structures. With proper restrictions, the resulting partial differential equation (PDE) are elliptic in equilibrium problems. Due to assumptions made in classical CM, see section 2, this theory is unable to properly model damage evolution after initiation $[5,6]$. To overcome this deficit, additional theories, such as fracture mechanics, are required. However, the majority of these 
techniques still suffer from unsatisfactory accuracy and low efficiency when dealing with specific problems, such as three-dimensional crack propagation in bodies under complex loading conditions [7].

Motivated by ideas of molecular dynamics, Stewart Silling developed the peridynamic theory in the early 2000's as an alternative theory to classical CM [6]. The peridynamic (PD) theory is a promising approach to simulate damage initiation, evolution and interaction in any material. It is a non-local theory taking long-range forces between material points in a certain neighborhood into account. In this theory the divergence term of the governing PDE is replaced by an integral term. Constitutive models in PD depend on relative deformation vectors as opposed to classical constitutive models, depending on deformation gradients [8]. In addition, spatial derivatives in the governing equations of classical CM are replaced by integral terms in PD. Theses, integral terms can be evaluated at any point whether a discontinuity in the displacement function, e.g. caused by a crack, exists or not. The original bond-based peridynamics (BB-PD) formulation has been introduced by Silling [6] and is based on the interaction forces between two material points. Therein, linear isotropic materials are limited to a Poisson ratio of 0.25 for $3 \mathrm{D}$ and $2 \mathrm{D}$ plane strain problems and 0.33 for $2 \mathrm{D}$ plane stress problems [9]. To overcome these limitations, Silling et al. [9] introduced a state-based peridynamics (SB-PD) formulation. It is based on the collective interaction forces between a given material point and material points in a finite neighborhood of this point, called family $\mathcal{H}$. In this paper, it is assumed that these forces act along the direction vectors between the material points near the natural state. This is called ordinary state-based (OSB) and considers bond forces depending on deformations of all neighboring material points. The state-based PD is able to describe materials with arbitrary Poisson's ratios [9].

Silling et al. [10] compare the classical CM and the PD for the conservation of linear momentum. The classical CM uses a differential equation, whereas the PD uses an integral formulation

$$
\rho(\mathbf{x}) \ddot{\mathbf{u}}(\mathbf{x}, t)=\operatorname{div}(\boldsymbol{\sigma})+\mathbf{b}(\mathbf{x}, t),
$$

with $t, \mathbf{b}, \rho, \boldsymbol{\sigma}$ and $\ddot{\mathbf{u}}$ as the time, the external forces, the mass density, the Cauchy stresses and the acceleration for the position $\mathbf{x}$. If a problem described by classic CM has a discontinuity, the model assumptions for this region are no longer valid. The classical CM loses its validity, as $\operatorname{div}(\boldsymbol{\sigma})$ is no longer defined. Therefore, the classical CM is no longer able to provide physically meaningful solutions. This vadility problem does not exist in PD. The integral formulation ensures the conservation of linear momentum at any point, even at discontinuities. No model adjustments or extensions need to be made. If the medium of the integration domain, is continuous and the deformation is twice continuously differentiable for $\lim _{\mathcal{H} \rightarrow 0}$, the PD solution converges to the classical CM solution [11].

In order to model damage initiation and propagation in PD theory, failure criteria are required ideally referring to physically measurable values. These criteria are needed to assess whether individual bonds, interactions of points within $\mathcal{H}$, have exceeded their load-bearing capacity. The most common criterion used in PD compares the relative change of distance, the so called stretch, between two discrete points with a reference value, the critical stretch [12]. If the stretch between the two discrete points exceeds a certain value, the bond is irreversibly deleted. The damage starts and there is no load transfer via the broken bond. The critical stretch model works well for brittle material and has been validated for several problems $[13,14]$. However, the critical stretch is not a physically measurable material property. It is recalculated using assumptions from the PD theory and includes a discretization dependency [12]. Although bonds can break under shear deformation, this criterion only considers the volumetric part of the 
deformation, being insensitive to the deviatoric part. As a result, it is unsuitable to simulate the fracture observed in elasto-visco-plastic materials [15].

Foster et al. [1, 16] describe an energy-based failure criterion. The authors use a J-integral calculation at the crack tip to determine a critical bond energy density from the experimentally measurable energy release rate. The critical energy density of each bond is compared with this critical value. If the value is exceeded, the bond breaks. In the case of crack propagation, the sum of the local bond energy densities, related to the crack surface, leads back to the global value of the energy release rate.

Three energy based criteria considering mixed mode conditions and an implementation strategy are presented. The implementation is verified and a convergence study is performed. The implemented failure criteria are used to analyze the damage propagation in a micro structure model of a FRPs structure. The code as well as the models are published in [17] for further use.

\section{TheOreticAL BACKGROUND}

The ordinary state-based peridynamics (OSB-PD) is used to overcome the restrictions of the BB-PD. Foster et al. [1] noted, that within the state-based PD the word bond is used loosely. It only describes the relationship between two material points and can be abstractly thought of as an interaction potential. There is not necessarily a notion of direct connectivity, such as a spring-like force. To illustrate the later derivation for the energy criterion consistently and comprehensibly, the derivation of the linear elastic material law for OSB-PD is illustrated. The notation follows Silling et al. [9].

In contrast to spring-like forces, the magnitude of the force density vector states $\mid \underline{\mathbf{T}}[\mathbf{x}, t]\left\langle\mathbf{x}^{\prime}-\right.$ $\mathbf{x}\rangle|\neq| \underline{\mathbf{T}}\left[\mathbf{x}^{\prime}, t\right]\left\langle\mathbf{x}-\mathbf{x}^{\prime}\right\rangle \mid$ may not be equal for the OSB-PD formulation, cf. Figure 2 for the linear case with infinitesimal deformations. The equation of motion of the OSB-PD is

$$
\rho(\mathbf{x}) \ddot{\mathbf{u}}(\mathbf{x}, t)=\int_{\mathcal{H}}\left(\underline{\mathbf{T}}[\mathbf{x}, t]\left\langle\mathbf{x}^{\prime}-\mathbf{x}\right\rangle-\underline{\mathbf{T}}\left[\mathbf{x}^{\prime}, t\right]\left\langle\mathbf{x}-\mathbf{x}^{\prime}\right\rangle\right) \mathrm{d} V+\mathbf{b}(\mathbf{x}, t),
$$

where $\mathcal{H}$ is a spherical neighborhood of radius $\delta$, called horizon, centered at $\mathbf{x}$. All points $\mathbf{x}^{\prime}$ within the horizon of $\mathbf{x}$ are called family of $\mathbf{x}$. The force density vector state $\mathbf{T}$ maps the bond $\left\langle\mathbf{x}^{\prime}-\mathbf{x}\right\rangle$ to force per unit volume squared in the deformed configuration [9]. $\underline{\mathbf{T}}$ has to ensure the consistency with basic physical principles as the balance of linear momentum. To describe a material, constitutive models are needed. These models map specific deformation vector states $\underline{\mathbf{Y}}$ into the force density vector state $\underline{\mathbf{T}}$.

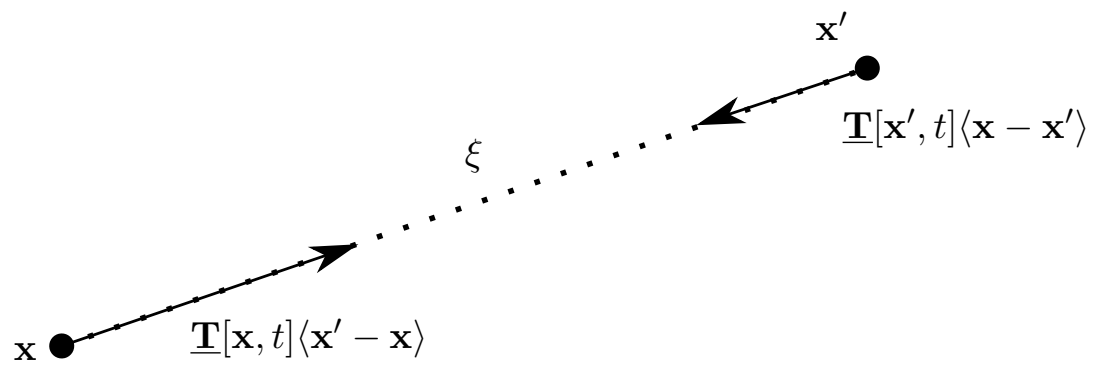

FiguRE 2. Illustration of OSB forces for the linear case with infinitesimal deformations.

Following Silling et al. [9] to determine the force density per volume square, for a PD material it is assumed that there exists a strain energy density function $W$ such that 


$$
\underline{\mathbf{T}}=\nabla W(\underline{\mathbf{Y}}),
$$

where $\nabla$ is the Fréchet derivative.

It is further assumed that the elastic strain energy in a PD solid is equal to the energy of the CM model. In that case, it is supposed that there is a PD strain energy density function $W: V \rightarrow \mathbb{R}$ such that, for some choice of the deformation gradient $\mathbf{F}$

$$
\underline{\mathbf{Y}}(\boldsymbol{\xi})=\mathbf{F} \boldsymbol{\xi}=\mathbf{F}\left\langle\mathbf{x}^{\prime}-\mathbf{x}\right\rangle \quad \forall \boldsymbol{\xi} \in \mathcal{H} .
$$

Then the PD corresponds to the classical constitutive model. This means that the classical CM and the PD model are identical for homogeneous deformations of a homogeneous body with deformation gradient $\mathbf{F}[9,18]$.

For the isotropic case and considering infinitesimal deformations the undeformed scalar state is defined as $\underline{x}=|\underline{\mathbf{X}}\langle\boldsymbol{\xi}\rangle|$ and the deformation scalar state is defined as $y=|\underline{\mathbf{Y}}\langle\boldsymbol{\xi}\rangle|$. Then, the extension scalar state $\underline{e}$ for a PD linear solid can be defined as

$$
\underline{e}=\underline{y}-\underline{x}=|\boldsymbol{\eta}|
$$

It must be noted that for a general material $\underline{y}-\underline{x} \neq|\boldsymbol{\eta}|$. To obtain the force density scalar state $\underline{t}=|\underline{\mathbf{T}}|$ of a single bond for an isotropic elastic PD solid, the extension scalar state is decomposed in its isotropic and deviatoric parts [9]. The decomposition of states utilizes a orthogonal deformation basis and therefore the isotropic and deviatoric deformation can be considered independently as

$$
\underline{e}^{d}\langle\boldsymbol{\xi}\rangle=\epsilon_{i j}^{d} \xi_{i} \frac{\xi_{j}}{|\boldsymbol{\xi}|} \quad \text { and } \quad \underline{e}^{i}\langle\boldsymbol{\xi}\rangle=\epsilon_{i i} \xi_{i} \frac{\xi_{i}}{|\boldsymbol{\xi}|} .
$$

Comparing classical CM strain energy with the PD material parameters based on the classical CM model are obtained. This is done by creating a strain energy function $W_{P D}$ using Equation 2-5 and comparing it with $W_{C M}$ as

$$
\begin{aligned}
W_{C M} & =\frac{1}{2} K\left[\epsilon_{k k}\right]^{2} \delta_{i j}+2 G\left[\epsilon_{i j}^{d}\right]^{2} \stackrel{!}{=} W_{P D} \\
W_{P D} & =\frac{A}{2} \int_{\mathcal{H}} \underline{\omega}\langle\boldsymbol{\xi}\rangle\left[\epsilon_{i j}^{d} \xi_{i} \frac{\xi_{j}}{|\boldsymbol{\xi}|}\right]^{2} \mathrm{~d} V_{\boldsymbol{\xi}}+\frac{B}{2} \int_{\mathcal{H}} \underline{\omega}\langle\boldsymbol{\xi}\rangle\left[\epsilon_{i i} \xi_{i} \frac{\xi_{i}}{|\boldsymbol{\xi}|}\right]^{2} \mathrm{~d} V_{\boldsymbol{\xi}} .
\end{aligned}
$$

Because of the state decomposition, the unknowns $A$ and $B$ can be obtained solving the integrals and setting one deformation state to zero in each case. For $A$ and $B$ the following values can be determined

$$
A=\frac{3 K}{m_{V}} \quad \text { and } \quad B=\frac{15 G}{m_{V}} .
$$

Therein, $K$ and $G$ are the bulk and the shear modulus of classical theory of linear elasticity. Introducing $A$ and $B$ in Equation 2-6 the strain energy density of the PD then is

$$
W=\frac{3 K}{2 m_{V}} \int_{\mathcal{H}} \underline{\omega}\langle\boldsymbol{\xi}\rangle\left[\underline{e}^{i}\langle\boldsymbol{\xi}\rangle\right]^{2} \mathrm{~d} V_{\boldsymbol{\xi}}+\frac{15 G}{2 m_{V}} \int_{\mathcal{H}} \underline{\omega}\langle\boldsymbol{\xi}\rangle\left[\underline{e}^{d}\langle\boldsymbol{\xi}\rangle\right]^{2} \mathrm{~d} V_{\boldsymbol{\xi}} .
$$

with the weighted volume $m_{V}$ and the a non-local dilatation $\theta$ as

$$
m_{V}=\int_{\mathcal{H}} \underline{\omega}\langle\boldsymbol{\xi}\rangle \underline{x \underline{x}} \mathrm{~d} V_{\boldsymbol{\xi}} \quad \text { and } \quad \theta=\frac{3}{m_{V}} \int_{\mathcal{H}} \underline{\omega}\langle\boldsymbol{\xi}\rangle \underline{x e}\langle\boldsymbol{\xi}\rangle \mathrm{d} V_{\boldsymbol{\xi}}
$$


Using Equation 2-2, it is possible to derive the contribution of a single bond and its force density scalar state $\underline{t}$

$$
\underline{t}\langle\boldsymbol{\xi}, t\rangle=\frac{\underline{\omega}\langle\boldsymbol{\xi}\rangle}{m_{V}}\left[3 K \theta \underline{x}+15 G \underline{e}^{d}\right] .
$$

To obtain $\underline{\mathbf{T}}$, the force density scalar state has to be transformed from bond coordinates to global Cartesian coordinates. For small deformations and isotropic material the transformation can be done as

$$
\underline{\mathbf{T}}=\underline{t} \underline{\underline{\mathbf{Y}}} \mid \underline{\underline{\mathbf{Y}} \mid}
$$

The complete derivation is given in Silling et al. [9].

\section{Damage Model}

Foster et al. [1, 16] describe an energy-based failure criterion which is valid for state-based analysis. The criterion converts the globally measurable energy release rate into a local critical bond energy density. With the horizon $\delta$, the critical micro potential $w_{C}$ can be determined using the energy release rate $G_{0}$

$$
w_{C}=\frac{4 G_{0}}{\pi \delta^{4}} .
$$

If the bond micro potential is greater than this value, the bond is removed and an initial failure occurs. With the history-dependent scalar valued function $\chi(\boldsymbol{\xi}, t)$

$$
\chi(\underline{e}\langle\boldsymbol{\xi}\rangle, t)=\left\{\begin{array}{ll}
1 & \text { if } w(\underline{e}\langle\boldsymbol{\xi}\rangle)<w_{C} \\
0 & \text { otherwise }
\end{array},\right.
$$

the damage model can be included in Equation 2-10 as

$$
\underline{t}=\chi(\underline{e}\langle\boldsymbol{\xi}\rangle, t)\left(\frac{3 K \theta}{m_{V}} \underline{\omega x}+\frac{15 G}{m_{V}} \underline{\omega e^{d}}\right) .
$$

In an OSB formulation the bond energy can be determined as

$$
w=\int_{\boldsymbol{\eta}}\left(\underline{\mathbf{T}}[\mathbf{x}, t]\left\langle\mathbf{x}^{\prime}-\mathbf{x}\right\rangle-\underline{\mathbf{T}}\left[\mathbf{x}^{\prime}, t\right]\left\langle\mathbf{x}-\mathbf{x}^{\prime}\right\rangle\right) \mathrm{d} \boldsymbol{\eta}
$$

where

$$
\boldsymbol{\eta}=\mathbf{u}\left[\mathbf{x}^{\prime}, t\right]-\mathbf{u}[\mathbf{x}, t]
$$

is the bond displacement vector state. We recall from Figure 2 that $\underline{\mathbf{T}}[\mathbf{x}, t]\left\langle\mathbf{x}^{\prime}-\mathbf{x}\right\rangle$ and $\underline{\mathbf{T}}\left[\mathbf{x}^{\prime}, t\right]\left\langle\mathbf{x}-\mathbf{x}^{\prime}\right\rangle$ are bond force density vector states. As Figure 2 shows for an OSB model, the force density scalar states $\underline{t}$ depend on the direction $\left\langle\mathbf{x}-\mathbf{x}^{\prime}\right\rangle$ and $\left\langle\mathbf{x}^{\prime}-\mathbf{x}\right\rangle$. For an OSB formulation, the force density scalar state for the respective material point $\underline{t}[\mathbf{x}, t]$ and $\underline{t}\left[\mathbf{x}^{\prime}, t\right]$ has to be calculated. The distinction between contraction and extension is made by calculating the relative extension scalar state

$$
\underline{e}_{r e l}\langle\boldsymbol{\xi}\rangle=\frac{\underline{e}\langle\boldsymbol{\xi}\rangle}{\underline{x}}
$$


Thus, the bond is contracted if $\underline{e}_{r e l}\langle\boldsymbol{\xi}\rangle<0$ and extended if $\underline{e}_{r e l}\langle\boldsymbol{\xi}\rangle>0$. Different bond micro potentials could be applied, if needed. Because the decomposition of states is already done, additional bond energy criteria could be defined evaluating the isotropic compression $w_{c m p}$, the isotropic tension $w_{t e n}$ and the shear part $w_{s h r}$. The assumption behind this distinction is based on the crack modes defined within the classical fracture mechanics [19]. A mode I fracture is dominated by the tensile stress within the material and therefore only the isotropic part of the model has to be compared to the $G_{I C}$ energy. The same applies to the shear part $w_{s h r}$ of the model. This can be compared against the energy release rate $G_{I I C}$ from the shear-dominated mode II fracture type. The bond micro potential for compression and tension can be determined as

$$
w_{c m p}=w_{t e n}=0.25 \chi(\underline{e}\langle\boldsymbol{\xi}\rangle, t)\left(\frac{K[\mathbf{x}]}{m_{V}[\mathbf{x}]} \theta^{2}[\mathbf{x}, t]+\frac{K\left[\mathbf{x}^{\prime}\right]}{m_{V}\left[\mathbf{x}^{\prime}\right]} \theta^{2}\left[\mathbf{x}^{\prime}, t\right]\right) \underline{x}^{2},
$$

where the distinction between compression and tension can be made utilizing Equation 3-6; $\underline{e}_{r e l}\langle\boldsymbol{\xi}\rangle\left\langle 0\right.$ for contraction and $\underline{e}_{r e l}\langle\boldsymbol{\xi}\rangle>0$ for extension. The bond micro potential for the shear part is given as

$$
w_{s h r}=0.25 \chi(\underline{e}\langle\boldsymbol{\xi}\rangle, t)\left(B[\mathbf{x}]\left(\underline{e}\langle\boldsymbol{\xi}\rangle-\frac{1}{3} \theta[\mathbf{x}, t] \underline{x}\right)^{2}+B\left[\mathbf{x}^{\prime}\right]\left(\frac{1}{3} \theta\left[\mathbf{x}^{\prime}\right] \underline{x}-\underline{e}\langle\boldsymbol{\xi}\rangle\right)^{2}\right),
$$

where $B$ is given in Equation 2-7.

To evaluate the bond damage condition one of three criteria from Table 1 can be chosen.

The factor 0.25 results from the integration and the averaging of the bond force density state $\underline{t}$. This averaging is necessary, as the scalar force density is dependent on the direction [5]. The energy criterion, with

$$
w_{\text {bond }}=0.25 \chi(\underline{e}\langle\boldsymbol{\xi}\rangle, t)\left(\underline{t}[\mathbf{x}, t]-\underline{t}\left[\mathbf{x}^{\prime}, t\right]\right) \underline{e}\langle\boldsymbol{\xi}\rangle
$$

corresponds to the criterion from Foster et al. [1]. The power law criterion takes mixed mode fracture into account [20]. The separated energy criteria check the isotropic compression and tension energies as well as the shear energy separately. If critical values regarding the tension or shear energy are exceeded the bond breaks.

\begin{tabular}{lc}
\hline Name & Criterion \\
\hline Energy criterion & $w_{\text {bond }}>w_{C}$ \\
Power law & $w_{c m p}^{2}+w_{\text {ten }}^{2}+w_{\text {shr }}^{2}>w_{C, \text { cmp }}^{2}+w_{C, \text { ten }}^{2}+w_{C, \text { shr }}^{2}$ \\
Separated & $w_{c m p}>w_{C, \text { cmp }}$ \\
& $w_{\text {ten }}>w_{C, \text { ten }}$ \\
$w_{\text {shr }}>w_{C, \text { shr }}$ \\
\hline
\end{tabular}

TABLE 1. Three types of energy based damage criteria. 


\section{IMPLEMENTATION}

Peridigm is used in the context of the present study [21]. It is an open-source state-based PD code developed at Sandia National Laboratories for massively-parallel multi-physics simulations. Peridigm uses a finite element (FE) mesh as basis for its discretization. Hexahedron and tetrahedron elements are transformed into PD collocation points and associated with the respective element volume. The entire data structure is divided into PD collocation points and their neighbours within the horizon. In theory each point and its interaction with his neighbours could be calculated at a single computer core. Points with the same properties, material and damage model, are bundled into blocks.

In algorithm 1 the program structure for the explicit Verlet time integration, implemented in Peridigm, is shown. After initialization, a loop is started on the number of time increments $n_{\text {timeStep }}$. New displacements and forces at the collocation points are determined for each time step in the loop. These are then synchronized between the definition blocks and computer cores. This is required, because the time integration of the neighboring collocation points do not necessarily have to be performed on the same core as the collocation point itself.

After the data has been synchronized, a loop is started over all blocks $n_{\text {blocks }}$. Within this loop, all collocation points $n_{\text {nodes }}$ are looped twice. The first run calls the damage model routines and determines whether a bond between a collocation point and a neighbor within the family $n_{\text {neighbor }}$ must be broken. In the second loop, the force densities resulting from the interaction of every collocation point with its neighborhood are determined. The data is synchronized in a global vector and the Verlet time integration schema is performed.

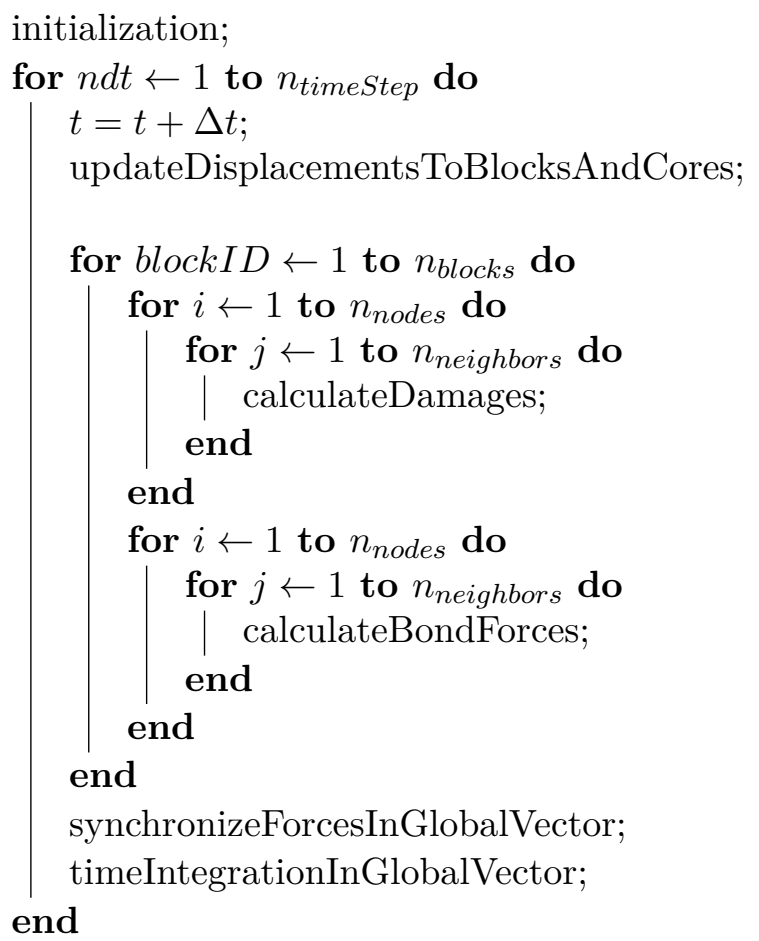

Algorithm 1: Peridigm data structure

For the implementation of the energy criterion, information from the collocation point $\mathbf{x}$ and $\mathbf{x}^{\prime}$ is required. Therefore, the solution shown in Figure 3 has been implemented in Peridigm. 


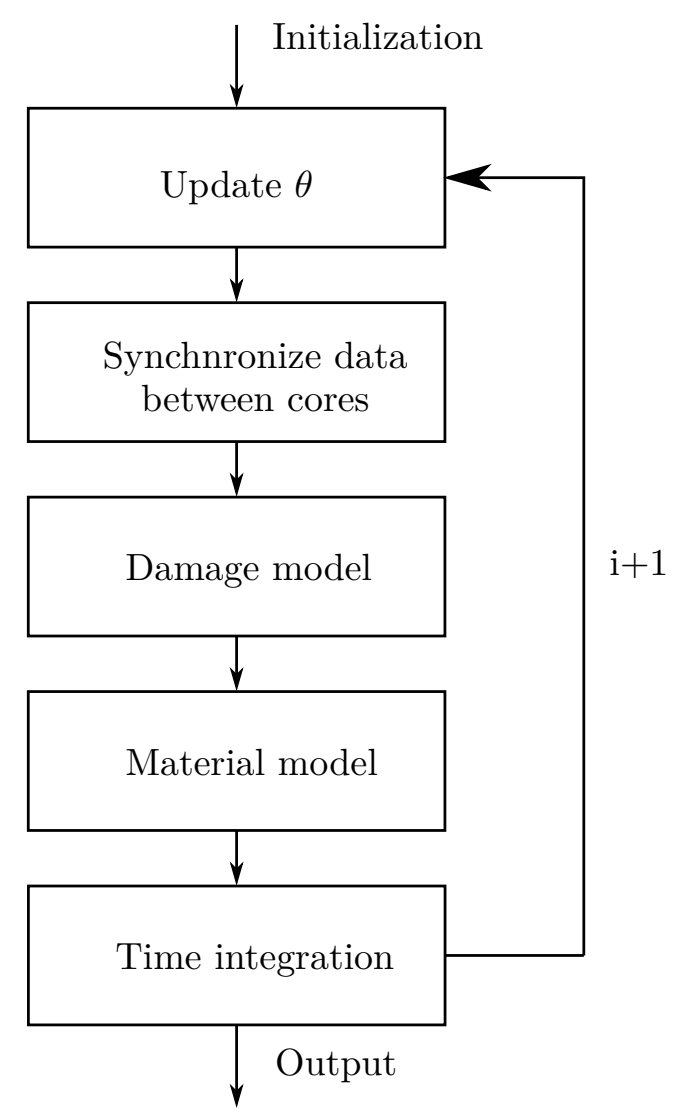

Figure 3. Workflow in Peridigm

For each step, the dilatation $\theta$ is calculated for the actual model deformation state $\mathbf{y}_{i}$. These dilatations are stored in a global vector and synchronized between the computer cores and blocks. In a next step, the force density scalar states in node $i$ and its neighbor $j$

$$
\begin{aligned}
\underline{t}_{i} & =\chi(\underline{e}\langle\boldsymbol{\xi}\rangle, t)\left(\frac{3 K_{i} \theta_{i}}{m_{V i}} \underline{\omega x}+\frac{15 G_{i}}{m_{V i}} \underline{\omega e}_{i}^{d}\right) \\
\underline{t}_{j} & =\chi(\underline{e}\langle\boldsymbol{\xi}\rangle, t)\left(\frac{3 K_{j} \theta_{j}}{m_{V j}} \underline{\omega x}+\frac{15 G_{j}}{m_{V j}} \underline{\omega e_{j}^{d}}\right)
\end{aligned}
$$

are determined. The bond micro potential then is

$$
w=0.25\left[\operatorname{abs}\left(\underline{t}_{i}\right)+\operatorname{abs}\left(\underline{t}_{j}\right)\right] \operatorname{abs}(\underline{e}\langle\boldsymbol{\xi}\rangle) .
$$

The absolute values of the force density scalar states as well as $\underline{e}\langle\boldsymbol{\xi}\rangle$ are used in the numerical implementation to avoid problems with coordinate changes between the node and neighbor node reference.

The volumetric part of the discrete bond micro potential for tension and compression can be calculated as 


$$
w_{t e n}=w_{c m p}=0.25 \chi(\underline{e}\langle\boldsymbol{\xi}\rangle, t)\left(\frac{K_{i}}{m_{V i}} \theta_{i}^{2}+\frac{K_{j}}{m_{V j}} \theta_{j}^{2}\right) \underline{x}^{2}
$$

and the deviatoric part as

$$
w_{s h r}=0.25 \chi(\underline{e}\langle\boldsymbol{\xi}\rangle, t)\left(\alpha_{i}\left(\underline{e}\langle\boldsymbol{\xi}\rangle-\frac{1}{3} \theta_{i} \underline{x}\right)^{2}+\alpha_{j}\left(\frac{1}{3} \theta_{j} \underline{x}-\underline{e}\langle\boldsymbol{\xi}\rangle\right)^{2}\right)
$$

with

$$
\alpha_{i}=\frac{15 G_{i}}{m_{V i}} \quad \text { and } \quad \alpha_{j}=\frac{15 G_{j}}{m_{V j}} .
$$

The damage laws have been implemented in Peridigm as an open-source routine [17].

\section{VERIFICATION}

Three problems are analyzed and evaluated for the validation of the proposed criteria and their implementation. The first problem is a virtual experiment used to determine the energy release rate. In this so-called double cantilever beam (DCB) experiment, a specimen with rectangular cross section, with an initial crack is loaded by pulling the upper and lower half. This triggers a controlled crack propagation from the initial crack initiation tip. The energy release rate can be determined by analyzing the force-displacement curves in relation to the crack length. The result of this evaluation must correspond to the input parameter in order to verify the assumptions made by Foster et al. [1]. Additionally, a convergence study is conducted. The horizon $\delta$ and the point distance $d x$ of a structured mesh are varied.

The example of a fracturing plate with a circular cutout under tensile loading is considered as a second problem. This model has an analytical solution in the context of classical linear elasticity [22] which yields to the position of the damage initiation. The PD solution is compared against an extended finite element method (XFEM) analysis performed in the commercial finite element code Abaqus.

5A. Double cantilever beam. The geometry and dimensions of the DCB model are given in Figure 4. The original finite elements are represented in Peridigm as points with corresponding volume. The numerical PD model has a structured discretization resulting in equally-spaced PD collocation points. The distance between all points is $d x=0.001 \mathrm{~m}$ for all three spatial directions.

A pre-crack of length $a_{0}$ is defined in the $x_{2}$-center of the model at the left specimen boundary. Bonds in the domain of the crack are omitted. Non-local displacement boundary conditions are applied to the left edges along $x_{3}$ to induce a crack opening. The displacement conditions are applied uniformly on more than one $x_{1}$ row of collocation points, $u_{2}^{+}(t)=0.004 \mathrm{~m} \mathrm{~s}^{-1} \cdot t$ above and $u_{2}^{-}(t)=-0.004 \mathrm{~m} \mathrm{~s}^{-1} \cdot t$ underneath the pre-crack. As a result, the upper part of the DCB model bends upwards and the lower part bends downwards. This results in a load concentration at the crack tip, which leads to a high bond micro potential. For an ideal homogeneous isotropic material, crack growth in $x_{1}$-direction occurs when the external load is further increased. The Python scripts for creating the model as well as the Peridigm input decks are given in [17].

To solve the problem, a transient analysis is performed. This requires time integration. For all the analyzes presented here, the explicit Verlet time integration scheme is used. The material parameters are shown in Table 2. An energy release rate of $G_{I C}=12 \mathrm{~N} \mathrm{~m}^{-1}$ is chosen. This value is not representative of any standard material and was chosen low to reduce the computing 


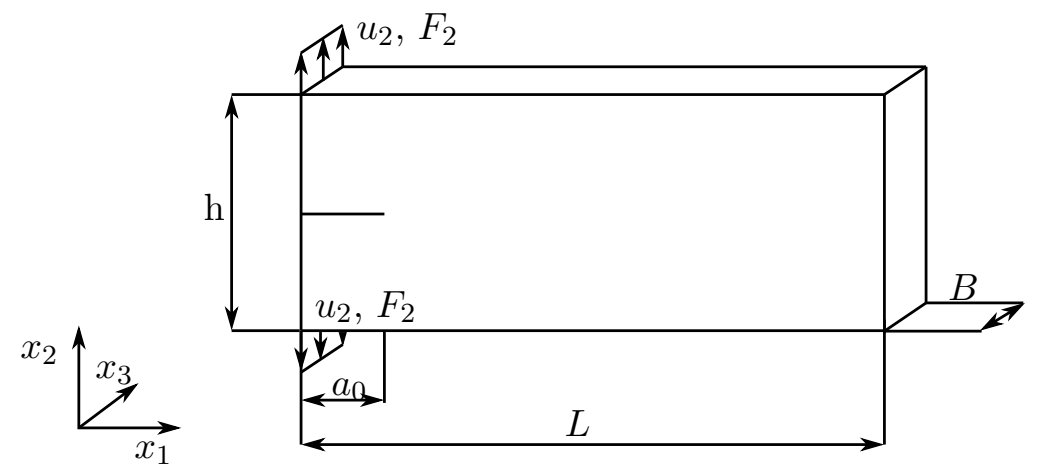

FigurE 4. Setup of the DCB simulation with $a_{0}=0.005 \mathrm{~m}, L=0.05 \mathrm{~m} B=0.006 \mathrm{~m}$ and $h=0.02 \mathrm{~m}$. The displacement function $u_{2}^{+}(t)=0.004 \mathrm{~m} / \mathrm{s} \cdot t$ above and $u_{2}^{-}(t)=$ $-0.004 \mathrm{~m} / \mathrm{s} \cdot t$ underneath the pre crack has been chosen.

time. The energy release rate determines the time of crack initiation and the degredation path after crack initiation.

In case the criteria are properly implemented, it must be possible to reproduce them in the virtual experiment, independent of the value itself.

\begin{tabular}{cccc}
\hline$\rho$ & $K$ & $G$ & $G_{I C}$ \\
{$\left[\mathrm{~kg} \mathrm{~m}^{-3}\right]$} & {$[\mathrm{Pa}]$} & $\begin{array}{c}\mathrm{Pa}] \\
{\left[\mathrm{N} \mathrm{m}^{-1}\right]}\end{array}$ \\
\hline 2000 & $1.75 \cdot 10^{9}$ & $8.08 \cdot 10^{8}$ & 12
\end{tabular}

TABLE 2. Material data chosen for the verification.

To determine the energy release rate $G_{I C}$, the force-displacement $\left(F_{2}(t), u_{2}(t)\right)$ curve are recorded and evaluated. The force function $F_{2}(t)$ is the summed resulting force of all nodes the displacement function $u_{2}(t)$ is applied on. The whole procedure is exemplary explained for the horizon $\delta=0.003 \mathrm{~m}$, shown in Figure 5. Prior to initial damage, the force-displacement curve starts with a linear response. This is the pre-damage part of the simulation. After damage initiation the resulting force decreases, while the crack propagates. To determine the resulting energy release rate $G_{I C}$, the dissipated energy $W_{\text {diss }}$ due to crack propagation within the material, is required.

Assuming no further energy dissipation occurs, the energy release rate can be determined by calculating the area between the force-displacement curve and a linear function. The linear function intersects the coordinate origin and the force-displacement curve of the model at an arbitrary time increment $n_{\text {cut }}$, shown as an example for one force-displacement curve and two linear function in Figure 5. It corresponds to an idealized relief path within the virtual DCB experiment. The area between the two curves represents the dissipated energy.

To integrate the area in-between the curves the Riemann sum

$$
W_{\text {diss }}=\Delta u \sum_{i=1}^{n_{\text {cut }}}\left(F_{i}-F_{i, \text { inear function }}\right)
$$

is determined. The energy release rate can be determined by dividing the dissipated energy $W_{\text {diss }}$ by the crack surface $A_{\text {crack }}=B l_{\text {crack }}$ as 


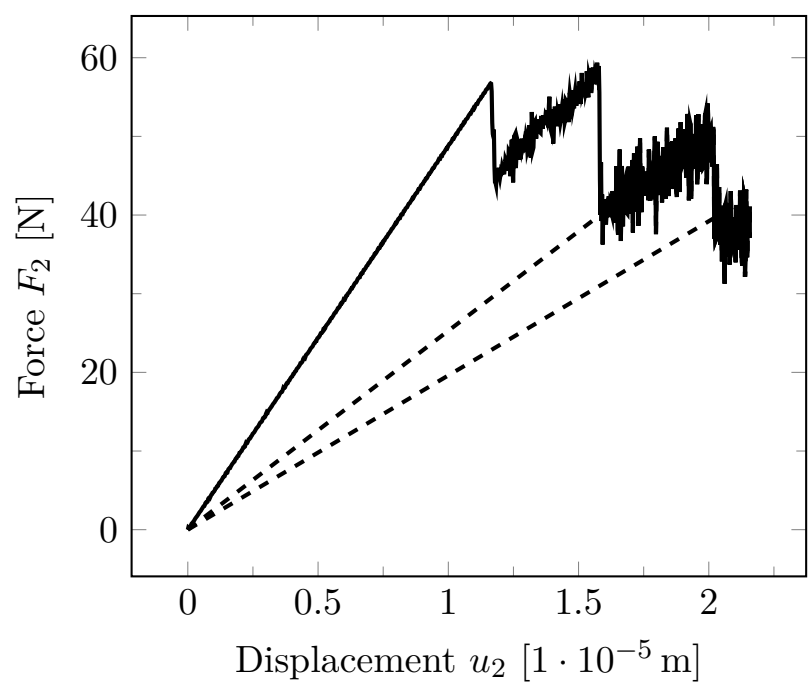

FIGURE 5. Force-displacement curve.

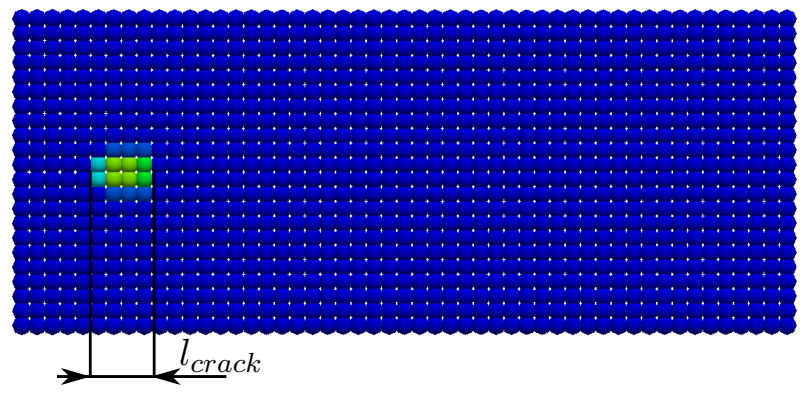

Figure 6 . Crack length at $t=2.21 \cdot 10^{-3} \mathrm{~S}$

$$
G_{I C}=\frac{W_{\text {diss }}}{B l_{\text {crack }}},
$$

where $l_{\text {crack }}$ is the crack length and $B$ is the width of the model [23]. To determine the energy release rate $G_{I C}$ for the increment $n_{\text {cut }}$ the corresponded crack length $l_{\text {crack }}$ is measured. For this purpose the damage index color map plots $I_{\text {damage }}$ for increment $n_{\text {cut }}$ is used, shown in Figure 6 for a point distance in all three spatial directions of $d x=0.001 \mathrm{~m}$. The damage index is determined as

$$
I_{\text {damage }}=n_{\text {broken }} / n_{\text {neighbors }},
$$

where $n_{\text {broken }}$ is the number of broken bonds of a point and $n_{\text {neighbors }}$ is the initial number of all of its bonds.

To show the independence of the horizon, four models with different horizons are calculated. To determine the energy release rate of the four models, two arbitrary linear functions are used for the respective curve similar to Figure 5. The time, the corresponding crack length and the resulting $G_{I C}$ are given in Table 3 . The force-displacement curves without the linear function for the DCB models with different horizons are shown in Figure 7. 


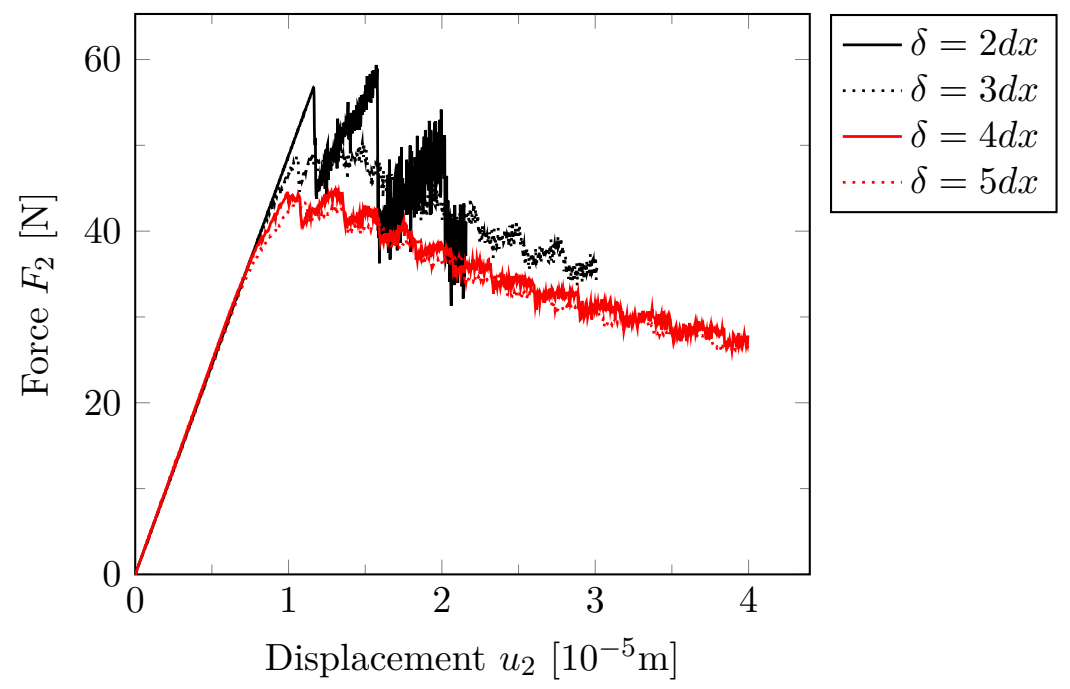

FiguRE 7. Force-displacement curves for $d x=0.001 \mathrm{~m}$.

\begin{tabular}{lccccccc}
\hline & \multicolumn{3}{c}{ Linear function 1} & \multicolumn{3}{c}{ Linear function 2} \\
Horizon $\delta$ & $\begin{array}{c}t \\
{[\mathrm{~m}]}\end{array}$ & $l_{\text {crack }}$ & $G_{I C}$ & $t$ & $l_{\text {crack }}$ & $G_{I C}$ \\
{$\left[10^{-3} \mathrm{~s}\right]$} & {$[\mathrm{m}]$} & {$\left[\mathrm{N} \mathrm{m}^{-1}\right]$} & {$[\mathrm{s}]$} & {$[\mathrm{m}]$} & {$\left[\mathrm{N} \mathrm{m}^{-1}\right]$} \\
\hline $2.015 \cdot 10^{-3}$ & 4.21 & 0.003 & 12.8 & 5.26 & 0.005 & 11.4 \\
$3.015 \cdot 10^{-3}$ & 5.88 & 0.005 & 13.1 & 7.35 & 0.007 & 12.9 \\
$4.015 \cdot 10^{-3}$ & 5.01 & 0.004 & 11.1 & 9.99 & 0.011 & 11.3 \\
$5.015 \cdot 10^{-3}$ & 6.36 & 0.006 & 11.2 & 9.99 & 0.010 & 11.9 \\
\hline
\end{tabular}

TABLE 3. Results of the verification.

Although the curves differ significantly from each other, the calculated energy release rates are well in line with the reference value of $G_{I C}=12 \mathrm{~N} \mathrm{~m}^{-1}$ defined in the Table 2. The differences in the results have three main reasons

- The crack length is rounded to $0.001 \mathrm{~m}$, which is the minimal distance between two nodes.

- The position of the crack tip is estimated using the damage index plot. This means that points that are not yet completely separated from each other would have a virtual crack length that cannot be clearly determined. The error is in the range $\pm 0.0005 \mathrm{~m}$. For a higher resolution this error becomes smaller.

- Due to the explicit time integration scheme without damping there is noise, which slightly influences the curve integration.

In conclusion, the results are accurate enough to demonstrate the functionality of the implemented failure models. The next step is to demonstrate the convergence of the model.

5B. Double cantilever beam - convergence. After the correct implementation is checked, the convergence behavior is analyzed. As described in section 2, the PD solution for homogeneous material converges against the classic CM solution in case no damage is considered. Therefore, 


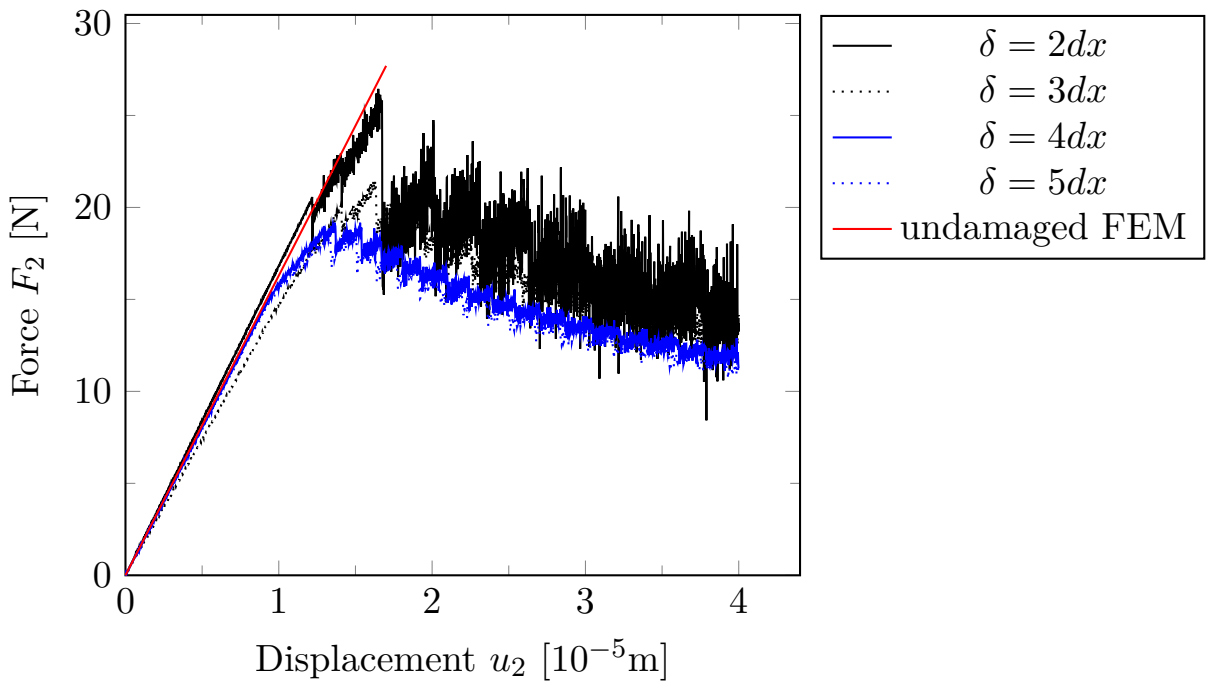

FiguRE 8. Force-Displacement curves for discretization $d x=0.5 \cdot 10^{-3} \mathrm{~m}$.

a model with linear elastic material behavior is created and solved with the FEM. The solution is used as a reference curve for the time before damage initiation. The geometry and material parameters are identical to the model data of the PD model.

The uniform point distance $d x$ in all three model dimensions of a structured mesh as well as the corresponding horizon $\delta$ is given in Table 4. Four different scenarios are considered: $d x=0.5 \cdot 10^{-3} \mathrm{~m}, d x=0.33 \cdot 10^{-3} \mathrm{~m}$, and $d x=0.25 \cdot 10^{-3} \mathrm{~m}$ and $d x=0.125 \cdot 10^{-3} \mathrm{~m}$. The model setup and material parameters are otherwise the same as in section $5 \mathrm{~A}$.

\begin{tabular}{|c|c|c|c|c|}
\hline$d x\left[10^{-3} \mathrm{~m}\right]=\delta$ & $2 d x\left[10^{-3} \mathrm{~m}\right]$ & $3 d x\left[10^{-3} \mathrm{~m}\right]$ & $4 d x\left[10^{-3} \mathrm{~m}\right]$ & $5 d x\left[10^{-3} \mathrm{~m}\right]$ \\
\hline 0.5 & 1.0 & 1.5 & 2.0 & 2.5 \\
\hline 0.3 & 0.66 & 0.99 & 1.32 & 1.65 \\
\hline 0.25 & 0.5 & 0.75 & 1.0 & 1.25 \\
\hline 0.125 & 0.25 & 0.375 & 0.5 & 0.625 \\
\hline
\end{tabular}

TABLE 4. Combinations for convergence analysis

Only the results of two cases are presented here, as they are very similar. The resulting curves described in Table 4 are shown in Figures 8 - 9. All discretizations $d x$ reflect the undamaged behavior until crack initiation compared to the FEM result. The time of crack initiation and the necessary forces differ considerably. All curves show that the highest force is required at a horizon of $2 d x$. The noise in the crack propagation area is also very high.

For the curves with a horizon of $3 d x$, the forces are also higher compared to the curves where the horizons are larger than $3 d x$. For horizons larger than $3 d x$, the crack initiation time and the force are approximately equal for the respective discretization $d x$. The behaviour in the crack propagation area is also identical. 


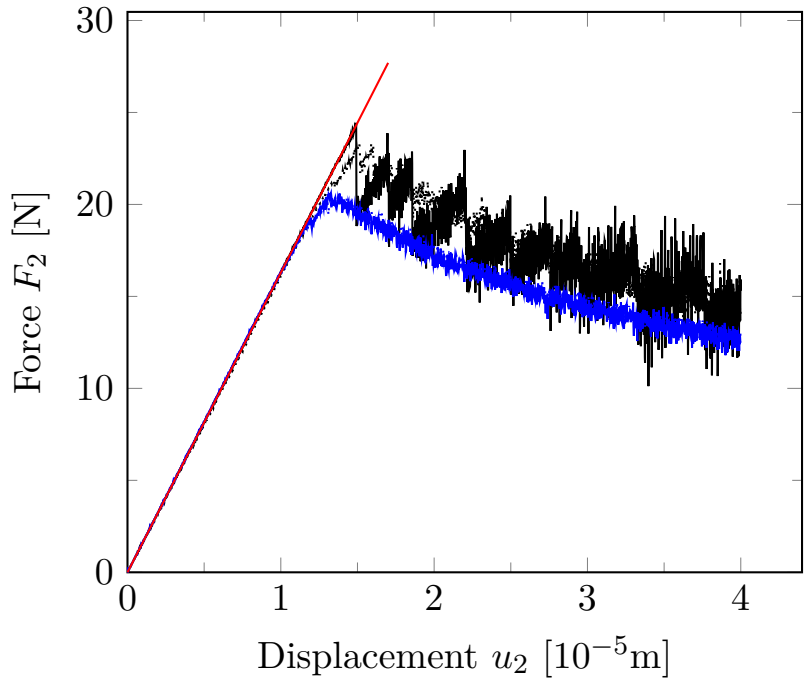

$$
\begin{aligned}
& -\quad \delta=2 d x \\
& \delta=3 d x \\
& \delta=4 d x \\
& \begin{array}{c}
\delta=5 d x \\
\ldots \text { undamaged FEM }
\end{array}
\end{aligned}
$$

Figure 9. Force-Displacement curves for discretization $d x=0.125 \cdot 10^{-3} \mathrm{~m}$.

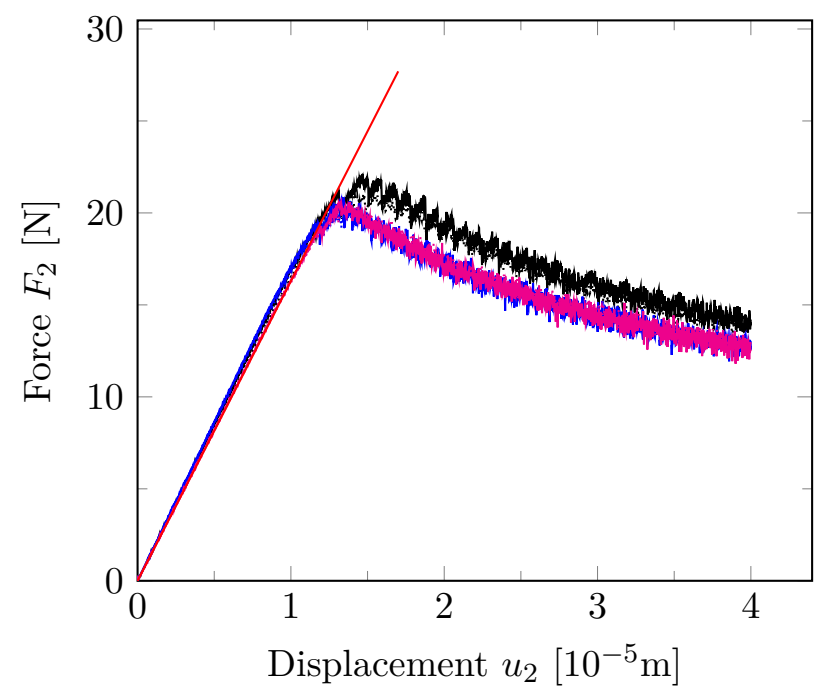

$$
\begin{gathered}
-d x=0.33 \cdot 10^{-3} \mathrm{~m}, \delta=4 d x \\
\cdots \cdots \cdot d x=0.33 \cdot 10^{-3} \mathrm{~m}, \delta=5 d x \\
-d x=0.25 \cdot 10^{-3} \mathrm{~m}, \delta=4 d x \\
\cdots \cdots \cdot d x=0.25 \cdot 10^{-3} \mathrm{~m}, \delta=5 d x \\
-d x=0.125 \cdot 10^{-3} \mathrm{~m}, \delta=4 d x \\
\cdots \cdots \cdot d x=0.125 \cdot 10^{-3} \mathrm{~m}, \delta=5 d x \\
\quad \text { undamaged FEM }
\end{gathered}
$$

Figure 10. Force-Displacement curves for horizons $4 d x$ and $5 d x$ for various discretizations $d x$.

To analyze the discretizations, the curves $4 d x$ and $5 d x$ of the discretization $d x=0.33 \cdot 10^{-3} \mathrm{~m}$, $0.25 \cdot 10^{-3} \mathrm{~m}$ and $0.125 \cdot 10^{-3} \mathrm{~m}$ are plotted in Figure 10 .

The results of the three discretizations show the same behavior in the linear pre-crack part of the simulation. After crack initiation the resulting forces for both horizons $4 d x$ and $5 d x$ of $d x=0.33 \cdot 10^{-3} \mathrm{~m}$ are $10-15 \%$ higher compared to the models with the discretization of $d x=0.25 \cdot 10^{-3} \mathrm{~m}$ and $d x=0.125 \cdot 10^{-3} \mathrm{~m}$. These curves are almost identical. They show the same, converged, damage initiation load of $20.5 \mathrm{~N}$ and the degredation path is similar.

In general, for horizons $\delta>3 d x$ the curves after crack initiation are smoother. The reason is, that for smaller horizons one bond caries more load, which leads to more noise if the bond breaks. This effect is smaller for larger horizons. 
5C. Plate with hole. After the convergence of the PD energy criterion has been demonstrated a plate with a circular hole under tensile loading is analyzed.

The classical CM has analytical solutions for the stress distribution around the hole [22], which have been validated in experiments. This allows to predict the location of damage initiation. The crack is assumed to emerge from points on the boundary of the cutout perpendicular to the loading direction. This is due to the fact, that the maximum stress concentration under tensile loading occurs at these points in CM. Furthermore, the crack is predicted to propagate horizontally, transverse to the loading direction.

Additionally, the results are compared with a XFEM fracture model consisting of an initiation criterion and a propagation model. The standard FEM does not allow crack propagation through elements. It is only capable to represent crack growth by numerically expensive adaptive remeshing techniques around the crack front or the element deletion method, where energy is artificially dissipated from the model. An enhancement of the FEM is the XFEM which offers a possibility to simulate crack growth through the element domain by application of the "partition of unity" approach $[24,25]$. The commercial FE code Abaqus offers an implementation of the XFEM.

For both the PD and XFEM analysis, a three-dimensional rectangular plate made of homogeneous, isotropic and linear elastic material with a circular cutout in its center is considered. The geometry of the plate is shown in Figure 11. The plate has a length and width of $L=M=0.05 \mathrm{~m}$. The thickness of the plate is $h=0.5 \cdot 10^{-3} \mathrm{~m}$. The hole diameter is $D=0.01 \mathrm{~m}$.

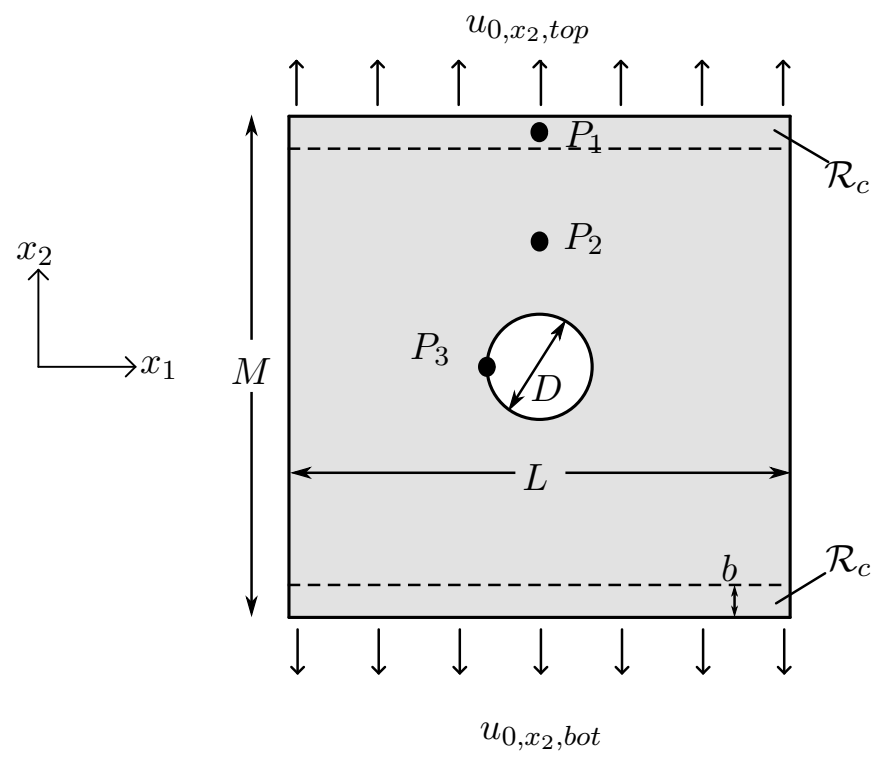

Figure 11. Model definition for a plate with a circular hole in its center $L=M=$ $0.05 \mathrm{~m}, h=0.5 \cdot 10^{-3} \mathrm{~m}$ and $D=0.01 \mathrm{~m}$

The material properties are given in Table 5. The model is calculated using the energy criterion proposed by Foster et al. [1]. The boundary conditions $\dot{u}_{y, t o p}$ and $\dot{u}_{y, b o t}$, the horizon $\delta$ and the average distance between mesh points $d x$ are given in Table 6 .

Peridigm is sensitive to the horizon size. The spacing between material points should be chosen uniformly in one block in order to reduce the effect of discretization errors. Thus, the 


\begin{tabular}{ccccc}
\hline $\begin{array}{c}\rho \\
{\left[\mathrm{kg} \mathrm{m}^{-3}\right]}\end{array}$ & $\begin{array}{c}E \\
{[\mathrm{~Pa}]}\end{array}$ & $\begin{array}{c}\nu \\
{[-]}\end{array}$ & $\begin{array}{c}G_{I C} \\
{\left[\mathrm{~N} \mathrm{~m}^{-1}\right]}\end{array}$ & $\begin{array}{c}s_{C} \\
{[-]}\end{array}$ \\
\hline 8000 & $192 \cdot 10^{6}$ & 0.33 & $287.072 \cdot 10^{3}$ & 0.02 \\
\hline
\end{tabular}

TABLE 5. Material data chosen for a plate with hole.

underlying mesh has to consist of approximately uniformly spaced elements. The requirement of generating constant mesh elements of length $d x=0.5 \cdot 10^{-3} \mathrm{~m}$ is given to the mesh generator. This results in a constant horizon size of $\delta=3.015 \cdot d x=0.15075 \cdot 10^{-3} \mathrm{~m}$.

To trigger damage, the plate is subjected to a constant uniaxial velocity along the horizontal edges in opposite direction, as illustrated in Figure 11.

\begin{tabular}{ccccc}
\hline $\begin{array}{c}\dot{u}_{y, t o p} \\
{\left[\mathrm{~m} \mathrm{~s}^{-1}\right]}\end{array}$ & $\begin{array}{c}\dot{u}_{y, \text { bot }} \\
{\left[\mathrm{m} \mathrm{s}^{-1}\right]}\end{array}$ & $\begin{array}{c}\delta \\
{[\mathrm{m}]}\end{array}$ & $\begin{array}{c}t_{\text {end }} \\
{[\mathrm{s}]}\end{array}$ & $\begin{array}{c}d x \\
{[\mathrm{~m}]}\end{array}$ \\
\hline 0.27541 & -0.27541 & $1.515 \cdot 10^{-3}$ & 0.001 & 0.0005 \\
\hline
\end{tabular}

TABLE 6. Model parameters.

The boundary conditions are applied to two constrained regions $\mathcal{R}_{c}$. They are defined along the bottom and top horizontal edges of the plate, as illustrated in Figure 11. Both regions have a depth of $b=3 \cdot d x$. The imposed displacements are given by the prescribed velocities $\dot{u}_{x_{2}, t o p}$ for the top part and $\dot{u}_{x_{2}, \text { bot }}$ for the bottom part, each multiplied by the current calculation time. This results in a uniformly increasing displacement over time. Thus, the prescribed displacement applied on each PD material point in the top constrained region is given by $u_{0, x_{2}, \text { top }}=\dot{u}_{x_{2}, t o p} \cdot t$ and in the bottom constrained region is given by $u_{0, x_{2}, b o t}=\dot{u}_{x-2, b o t} \cdot t$. The prescribed velocities, the horizon $\delta$ and the time $t_{\text {end }}$ are given in Table 6.

The XFEM analysis has been performed on a two-dimensional model utilizing the commercial code Abaqus. The thickness is considered irrelevant for the present plane-stress problem. For the application of the XFEM an extra damage initiation criterion is needed.

This assumption is necessary to change the underlying model within the FEM. Until damage occurs, the modeling of the FEM and XFEM is based on the approximate solution of the weak formulation of classical CM. After the crack has been initiated, the underlying assumptions lose their validity locally. Therefore, a fracture mechanical model describing the crack propagation within the XFEM elements is used. The maximum principal strain criterion is used as the initiation criterion. The strain value has been calculated using the energy release rate $G_{0 C}$ as provided by Madenci [12] for 3D models

$$
\varepsilon_{C}=s_{C}=\sqrt{\frac{G_{0 C}}{\left[3 G+\left(\frac{3}{4}\right)^{4}\left(K-\frac{5 G}{3}\right)\right] \delta}} .
$$

The assumption is only acceptable as long as no shear strain occurs in the known area of crack initiation. At the position of crack initiation the strain $\varepsilon_{22}$ is approximately equal to the critical stretch. However, this assumption is not generalizable. A consistent derivation of the critical stretch from the energy release rate is not possible for OSB-PD [26]. If the principal strain given 
by Equation 5-4 exceeds the value, the crack starts and a bilinear traction separation law as illustrated in [27] is used. The XFEM uses a quasi-static analysis. To avoid singularities in the stiffness matrix a viscous damping is introduced.

For the XFEM model, damage initiation occurs at time $t=0.672 \mathrm{~ms}$ for a total displacement of $u_{y}=0.370 \mathrm{~mm}$. The crack initiation and propagation is shown in a damage index plot in Figure 12.

In Figure 12 (b), when the first damage is visible, the critical stretch value has been reached. In order to reach complete failure of an element, the critical separation has to reach the value $\Delta_{\text {fail }}$, which is indicated by red elements in the damage plot. Note that the damage variable used in the damage plots for XFEM has a different definition compared to the damage plots for the PD solution. In Figure 12, blue color indicates undamaged elements, equivalent to entirely undamaged bonds of a material point in Peridigm. Red elements in an Abaqus damage plot indicate that an element has failed and a crack runs through it. In that case the element has a local damage value of 1 . The damage index in Peridigm, given in Equation 5-3, describes the ratio of broken to initially unbroken bonds within the family of a collocation point. If a crack occurs between two points, they are still connected with their neighboring nodes at their corresponded sites of the crack. For the definition of a complete fracture plane, comparable to the DCB model, a local damage value of approximately 0.41 is sufficient. The PD collocation point then is completely disconnected from his neighbors at the other site of the crack surface.

It can be seen in Figure 12 that the crack growth on both sides of the hole is not proceeding uniformly. This might be due to non-uniformity in the mesh. Thus, the crack growth behaves slightly different on both sides of the plate. The effect is especially noticeable for the propagation of the crack on the right side of the cutout from time $t=0.7731 \mathrm{~ms}$ (Step 1273) to $t=0.8264 \mathrm{~ms}$ (Step 1800), as seen in Figure $12(\mathrm{l})-(\mathrm{m})$. During this period, the crack on the right is not propagating further while the crack on the left side has already reached the left boundary of the plate. For the XFEM the unsymmetric mesh leads to a stagnation of the crack at one side and finally to an unsymmetric solution. In theory, this should not be the case for a perfectly homogeneous structural model without numerical inaccuracies in the FEM and the symmetric load application as well as the chosen boundary conditions. In contrast to the XFEM, the impact of the discretization to the result is lower for the PD result. This is because, PD is implemented as a meshless method in Perdigim, which is independent of the composition of the mesh. Therefore, this can be seen as an advantage of PD compared to XFEM.

In the Abaqus computation total failure occurs at time $t=0.872 \mathrm{~ms}$ with a total displacement in $x_{2}$-direction of $u_{x_{2}, u l t}=0.456 \mathrm{~mm}$.

In Figure 13 the results obtained with Peridigm are shown. The damage initiation starts earlier compared to the XFEM solution. The crack propagates almost symmetrically in both directions. This is not caused by the underlying PD theory. Numerical implementation of PD problems similar to XFEM solution can occur.

Figure 14a and Figure 14b show the displacement curves at three points defined in Figure 11. Before damage initiation, the gradient of the three curves are equal. The PD result shows an earlier failure initiation compared to the XFEM analysis. The behaviour of the curves differ after crack initiation. This is caused by the use of different solving methods. Solving the XFEM model a quasi-static analysis is performed. The viscous damping in the XFEM solution decreases the noise in the displacement curves after the damage initiation occurs. The PD result uses an explicit time integration schema without damping. Thus the displacement solution shows oscillations. 
Damage variable

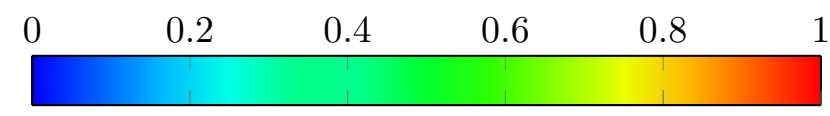

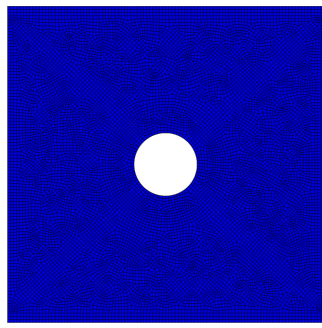

A) $t=0 \mathrm{~ms}$

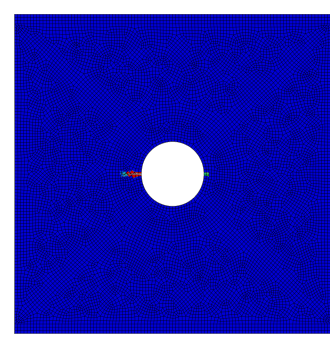

E) $t=0.773 \mathrm{~ms}$

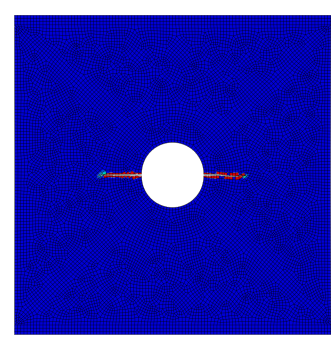

I) $t=0.773 \mathrm{~ms}$

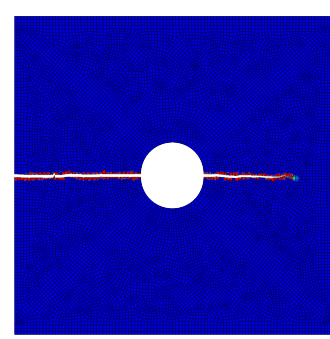

M) $t=0.82640 \mathrm{~ms}$

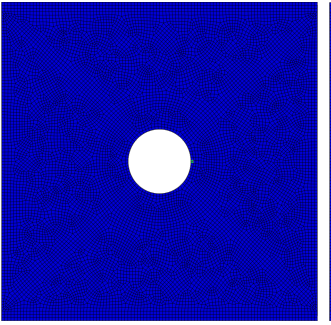

B) $t=0.67169 \mathrm{~ms}$

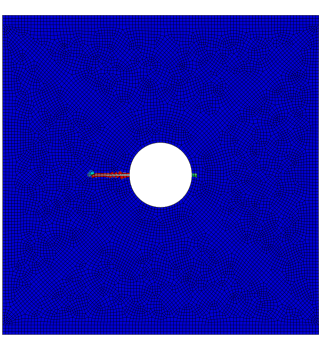

F) $t=0.773 \mathrm{~ms}$

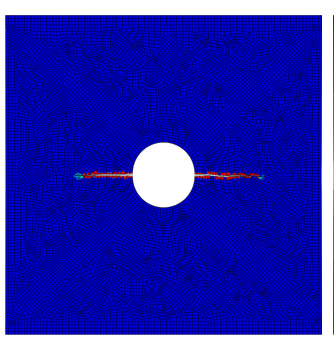

J) $t=0.77301 \mathrm{~ms}$

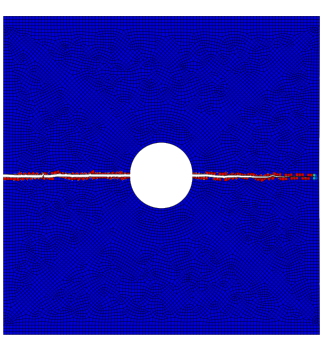

N) $t=0.82643 \mathrm{~ms}$

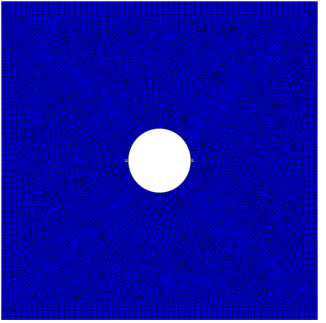

C) $t=0.67980 \mathrm{~ms}$

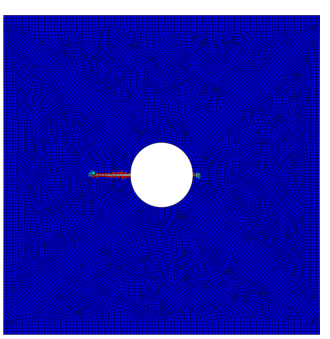

G) $t=0.773 \mathrm{~ms}$

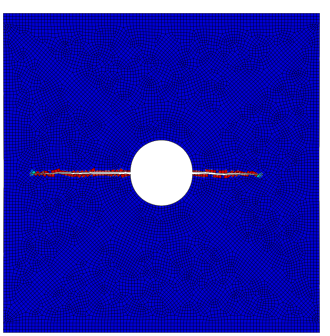

K) $t=0.77303 \mathrm{~ms}$

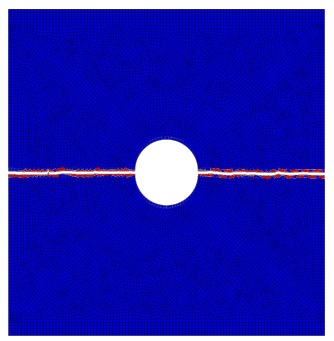

o) $t=0.82646 \mathrm{~ms}$

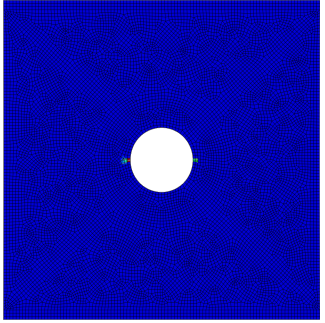

D) $t=0.773 \mathrm{~ms}$

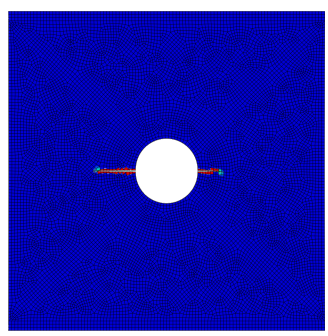

н) $t=0.773 \mathrm{~ms}$

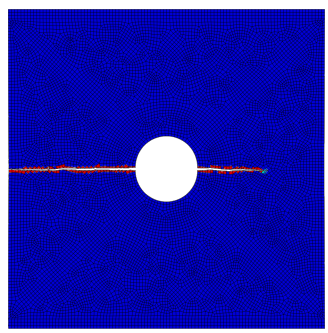

L) $t=0.77306 \mathrm{~ms}$

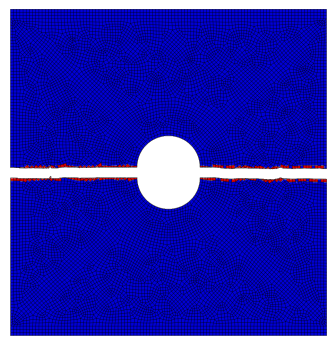

P) $t=3 \mathrm{~ms}$

Figure 12. Damage plot of plate with circular cutout under tensile loading for different time steps computed with XFEM. 
Damage variable

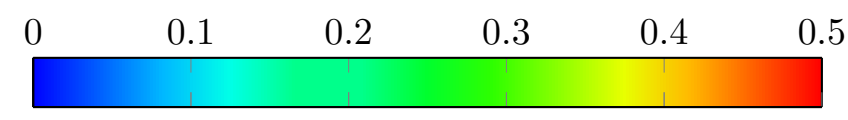

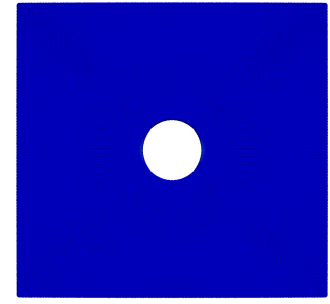

A) $t=0.63298 \mathrm{~ms}$

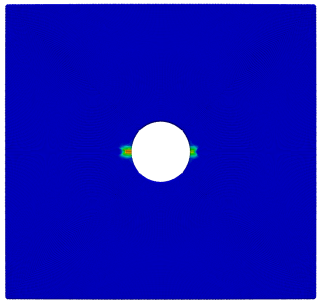

E) $t=0.69870 \mathrm{~ms}$

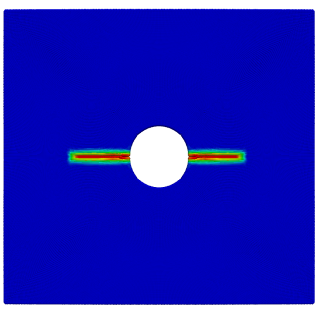

I) $t=0.70591 \mathrm{~ms}$

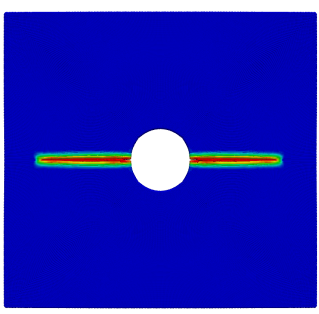

M) $t=0.70911 \mathrm{~ms}$

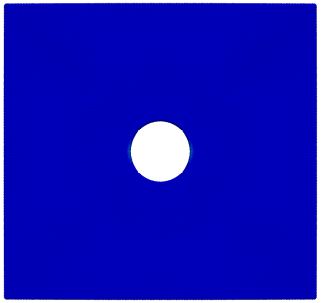

B) $t=0.6570 \mathrm{~ms}$

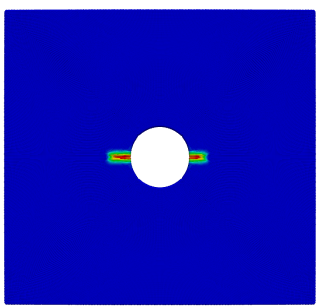

F) $\quad t$ $0.701104 \mathrm{~ms}$

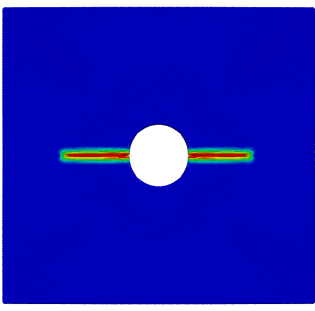

J) $t=0.70671 \mathrm{~ms}$

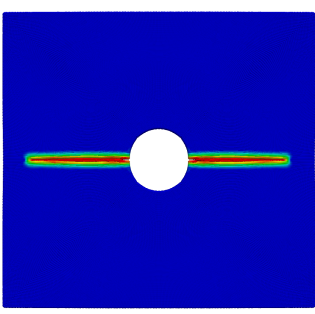

N) $t=0.70992 \mathrm{~ms}$

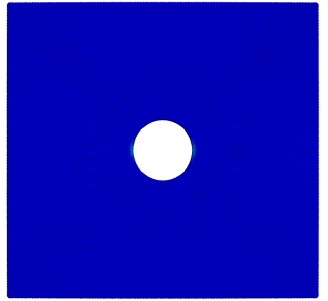

c) $t=0.673 \mathrm{~ms}$

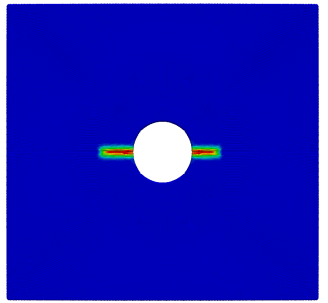

G) $t=0.70271 \mathrm{~ms}$

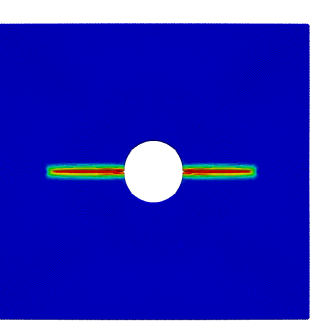

K) $t=0.70751 \mathrm{~ms}$

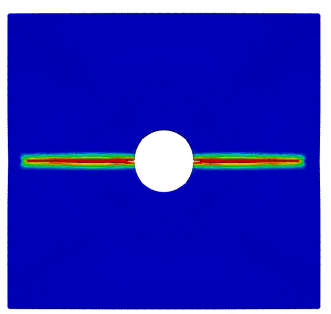

o) $t=0.71072 \mathrm{~ms}$

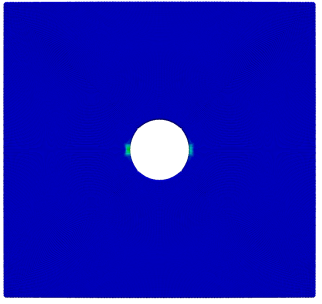

D) $t=$ $0.697097 \mathrm{~ms}$
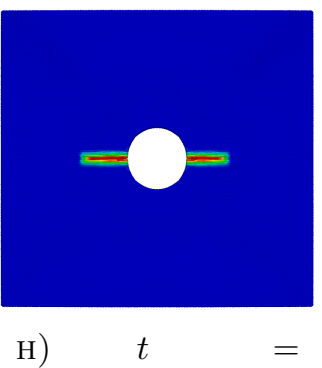
$0.704309 \mathrm{~ms}$

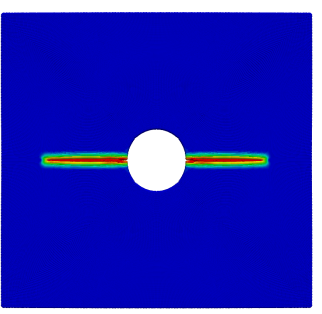

L) $t=0.70831 \mathrm{~ms}$

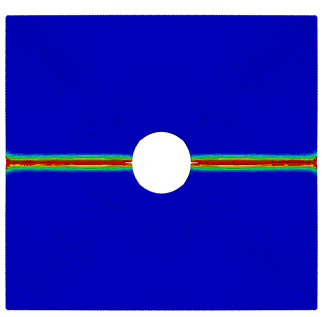

P) $t=0.71312 \mathrm{~ms}$

Figure 13. Damage plot of plate with circular cutout under tensile loading for different time steps computed with Peridigm. 


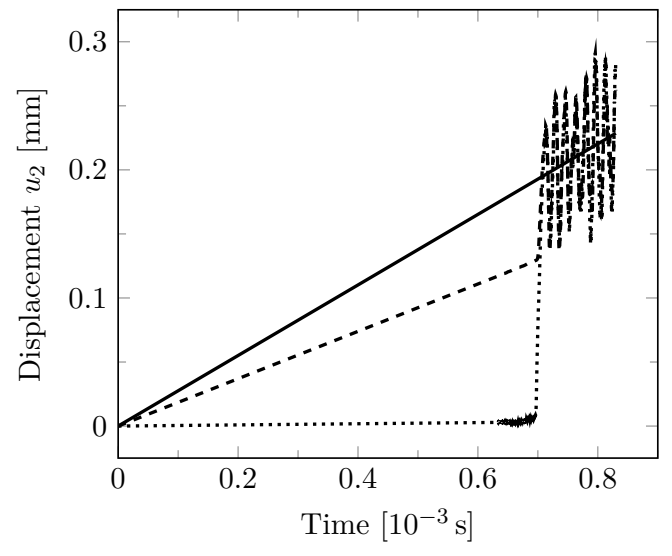

A) PD result.

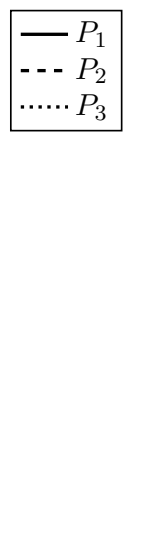

\section{.}

B) XFEM result with viscous damping of 0.0001 .

Figure 14. Comparison of displacement curves at given points $P_{1}, P_{2}$ and $P_{3}$, cf. Figure 11, for the Peridigm and XFEM model.

In summary, it can be said that the energy criterion is able to reproduce the expected crack initiation propagation. The following possibilities have been identified as reasons for the different initiation times

- Initiation criterion of XFEM differs from PD one.

- Dependency of the mesh in XFEM, which finally lead to an unsymmetrical crack.

- Step width and quasi-static solver of the XFEM analysis.

\section{Composite Modeling}

To study the effect of the different energy-based failure criteria, a scaled representative volume element (RVE) of height $h_{R V E}=0.025 \mathrm{~m}$ consisting of a FRP material has been analyzed. This model is based on the work of Krause [3, 28] and the fiber locations are randomly distributed. The model has been created utilizing a finite element pre-processor. The finite element input file, with the geometry and boundary conditions from Figure 15, has been automatically transformed into a input, readable by Peridigm.

The total model height is $h=h_{R V E}+2 h_{b}$. The height $h_{b}=0.00167 \mathrm{~m}$ is defined to ensure that the load introduced by the displacement function is equally distributed. The displacement function itself is applied at the top and bottom surface nodes of the RVE. Bonds within the boundary region cannot be damaged and the region is very stiff compared to the rest of the model.

Parameters for discretization were determined in Rädel et al. [29, 30] and used within the model to obtain accurate results. The horizon is given by $\delta=4 d x=0.002 \mathrm{~m}$ based on the analysis in subsection 5B and with $d x=5 \cdot 10^{-4} \mathrm{~m}$ as the average distance between two points, because of computational limitations. The material properties of both the fiber and the matrix made of resin are illustrated in Table 7 and the values for the different energy criteria are given in Table 8.

Figure 16 illustrates how the damage typically progresses in experiments under transverse tension. The cracks grow around single fibers, bridge the matrix-dominant area between fibers 


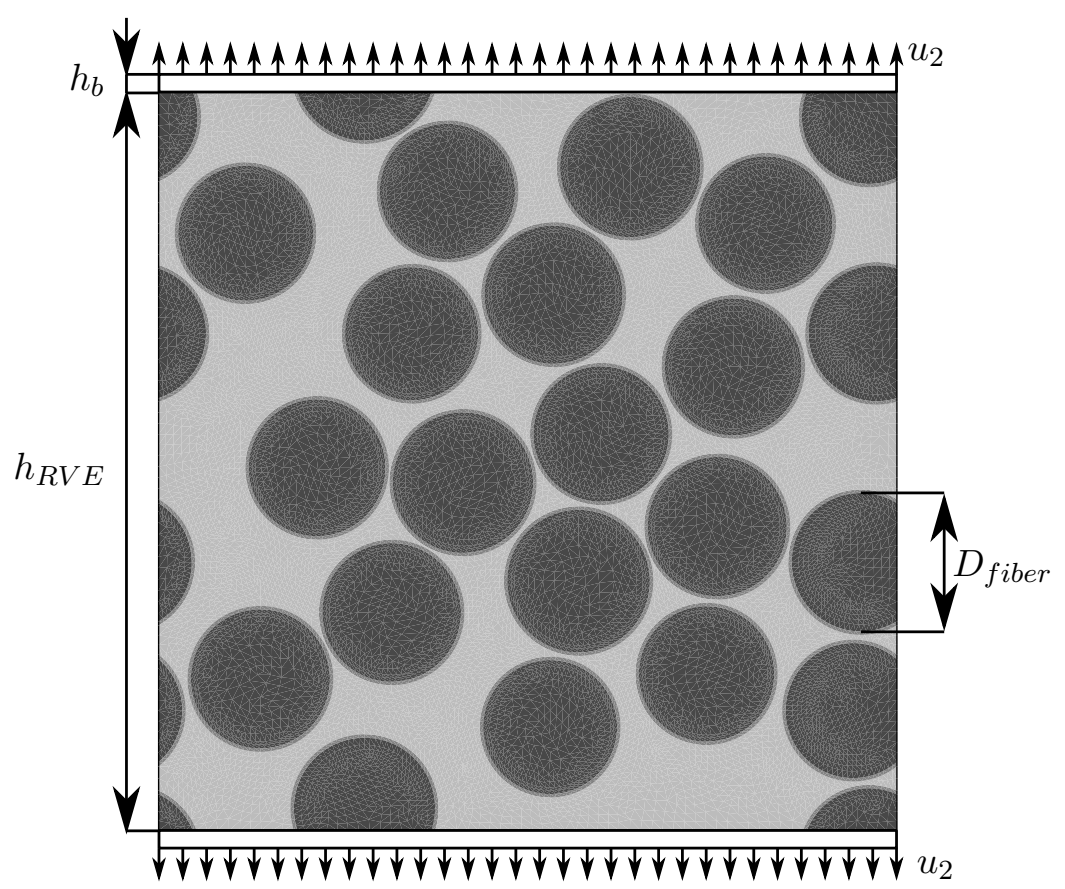

FIGURE 15. Setup of the RVE simulation $D_{\text {fiber }}=0.006744 \mathrm{~m}, h_{R V E}=0.025 \mathrm{~m}$ and $h_{b}=0.00167 \mathrm{~m}$. The displacement function is $u_{2}=0.213 \mathrm{~m} \mathrm{~s}^{-1} \cdot t$.

\begin{tabular}{cccc}
\hline Type & $\rho$ & $K$ & $G$ \\
& {$\left[\mathrm{~kg} \mathrm{~m}^{-3}\right]$} & {$\left[10^{6} \mathrm{~Pa}\right]$} & {$\left[10^{6} \mathrm{~Pa}\right]$} \\
\hline Fiber & 1800 & 17283 & 11382 \\
Resin & 1300 & 3125 & 1119 \\
\hline
\end{tabular}

TABLE 7. Material properties for the RVE.

\begin{tabular}{lccc}
\hline Type & $w_{\text {iso }}\left[10^{-3} \mathrm{~N} \mathrm{~m}^{-1}\right]$ & $w_{\text {cmp }}\left[10^{-3} \mathrm{~N} \mathrm{~m}^{-1}\right]$ & $w_{\text {ten }}\left[10^{-3} \mathrm{~N} \mathrm{~m}^{-1}\right]$ \\
\hline Energy criterion & 0.4 & - & - \\
Power law criterion & 0.4 & 0.4 & 0.38 \\
Separated energy criterion & 0.4 & 0.4 & 0.38 \\
\hline
\end{tabular}

TABLE 8. Critical energies chosen for the resin in the RVE model.

and finally interact with each other. Experimental and numerical results show that debonding occurs around many fibers but only one dominant transverse crack is finally created [3].

Figure 17 shows the results obtained by using the three energy based failure criteria, introduced in Table 1. Starting with the damage initiation, four different time steps are shown. It can be seen that the damage initiation as well as the ongoing damage process is almost equal between all criteria. The damage initiates at the fiber-matrix interface. The cracks grow and interact with each other. In the event of a further load increase, the various individual cracks 


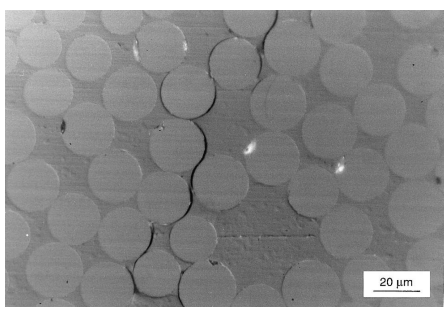

A) Debond

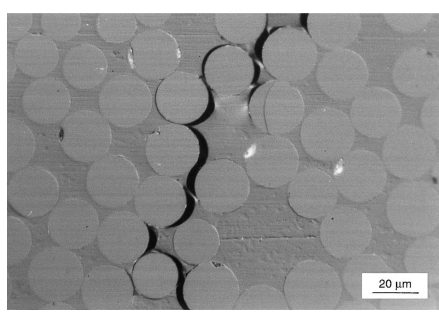

B) Transverse crack

Figure 16. Phases of damage under transverse tension [2]

combine to form an overall crack. The tensile part of the energy is dominant compared to the shear part. Thus the damage patterns of all models are similar. The crack patterns of the simulation results are in good agreement with the measurement results shown in Figure 1b. 


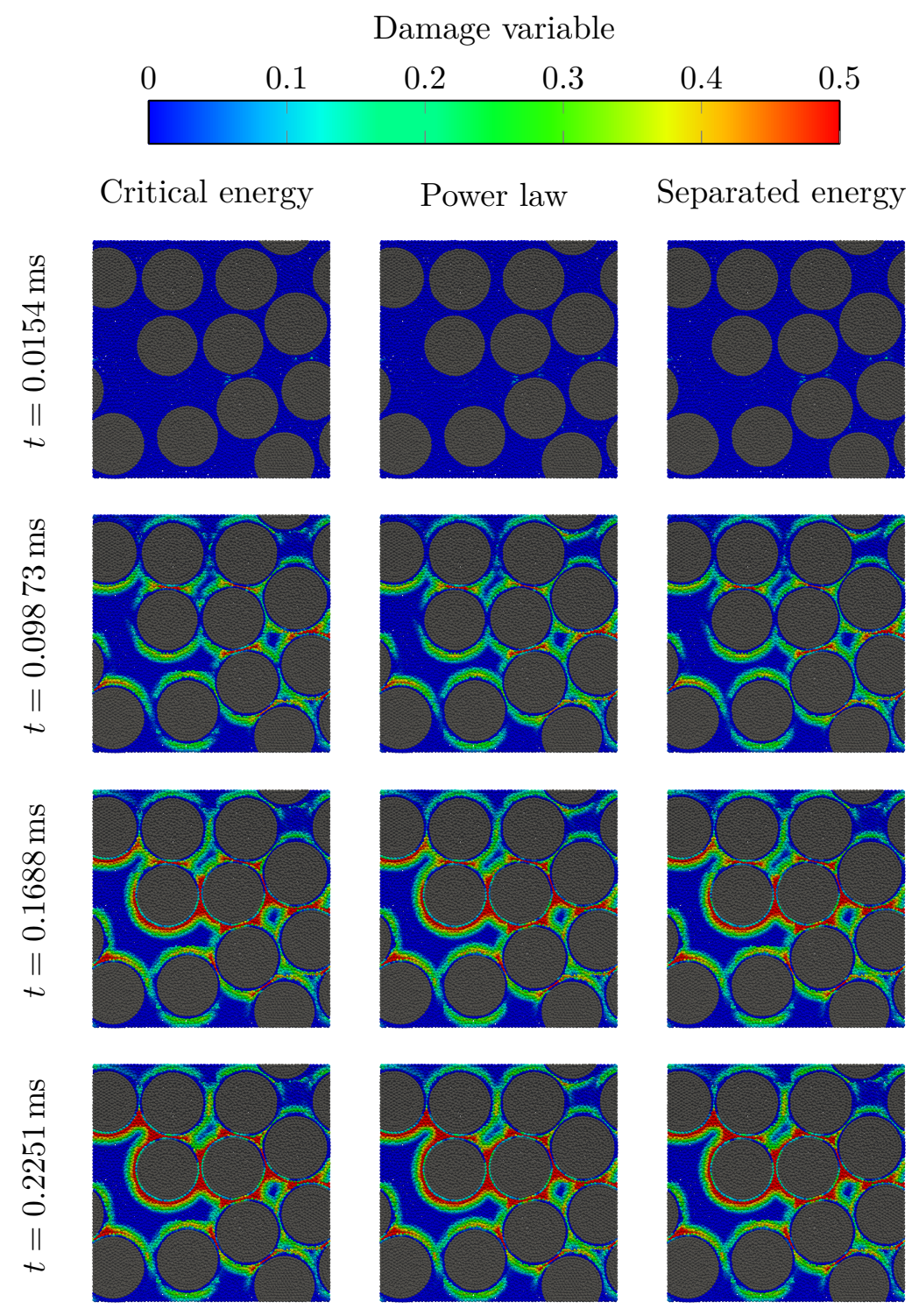

FIGURE 17. Damage plot of RVE utilizing the different energy criteria.

\section{Conclusion}

The implementation and verification of three energy based failure criteria have been presented. These criteria only require physically measurable material properties. The criteria have been implemented in the open-source code Peridigm. It is parallelizable and consequently usable for large scale problems. The verification examples indicate that the implemented criteria work as expected. It has been shown that global energy release rate could be represented by local micro energy bond potentials.

The model converges for a resolution $d x=0.25 \cdot 10^{-3} \mathrm{~m}$ with a horizon greater than $3 d x$. Regardless of the discretization, horizons smaller than $4 d x$ overestimate the crack initiation load and high noise in the damage progress area. 
The results of a complex micro structure model of a FRP illustrates that all implemented criteria are able to describe micro structural damage and the results do not differ substantially from literature results. The example illustrates that the general failure mechanism is captured by all three presented models. Therefore, the criterion of Foster et al. [1] is to be preferred. On the contrary to the two other criteria, only one material parameter, the energy release rate, has to be determined. In real applications, the experimental measurement of this parameter is already standardized and therefore easier to determine than for the other two criteria.

The final routine as well as the models are published within [17].

\section{REFERENCES}

[1] John T. Foster, Stewart A. Silling, and Weinong Chen. "An Energy based Failure Criterion for use with Peridynamic States". In: International Journal for Multiscale Computational Engineering 9.6 (2011), pp. 675-688. ISSN: 0020-7683. DOI: 10.1615/IntJMultCompEng . 2011002407. URL: http : / www . dl . begellhouse.com/ journals/61fd1b191cf7e96f , 5dbda3444284ba35, 7d22e9255b968290.html.

[2] E.K Gamstedt and B.A Sjögren. "Micromechanisms in tension-compression fatigue of composite laminates containing transverse plies". In: Composites Science and Technology 59.2 (1999), pp. 167-178. ISSN: 0266-3538. DOI: 10 . 1016/S0266-3538(98) 00061-X. URL: http://www.sciencedirect.com/science/article/pii/S026635389800061X.

[3] Daniel Krause. "Micromechanics of the fatigue behaviour of polymers". DLR Report 201626. PhD Thesis. Technical University Braunschweig, 2016.

[4] B. L. Boyce et al. "The Sandia fracture challenge: blind round robin predictions of ductile material tearing". In: International Journal of Fracture 186.1 (2014), pp. 5-68. ISSN: 15732673. DOI: 10 .1007/s10704-013-9904-6. URL: https ://doi .org/10 . 1007 /s10704013-9904-6.

[5] Florin Bobaru et al. Handbook of Peridynamic Modeling. Advances in Applied Mathematics. CRC Press, 2016. ISBN: 978-1315355948.

[6] Stewart A. Silling. "Reformulation of elasticity theory for discontinuities and long-range forces". In: Journal of the Mechanics and Physics of Solids 48.1 (2000), pp. 175-209. ISSN: 00225096. DOI: 10.1016/S0022-5096(99)00029-0.

[7] F. Mossaiby et al. "OpenCL implementation of a high performance 3D Peridynamic model on graphics accelerators". In: Computers \& Mathematics with Applications 74.8 (2017), pp. 1856-1870. ISSN: 0898-1221. DOI: $10.1016 / \mathrm{j}$. camwa . 2017.06 . 045. URL: http : //www.sciencedirect.com/science/article/pii/s0898122117304030.

[8] Pablo Seleson, Qiang Du, and Michael L. Parks. "On the consistency between nearestneighbor peridynamic discretizations and discretized classical elasticity models". In: Computer Methods in Applied Mechanics and Engineering 311 (2016), pp. 698-722. ISSN: 00457825. DOI: 10 . 1016 / j . cma . 2016 . 07 .039. URL: http : / /www . sciencedirect . com / science/article/pii/S0045782516308210.

[9] Stewart A. Silling et al. "Peridynamic States and Constitutive Modeling". In: Journal of Elasticity 88 (2007), pp. 151-184. DOI: 10.1007/s10659-007-9125-1.

[10] Stewart A. Silling. Linearized Theory of Peridynamic States. Sandia Report SAND20092458. Multiscale Science Department, Sandia National Laboratories, 2009. URL: http: //www. sandia.gov/ sasilli/SAND2009-2458.pdf.

[11] Stewart A. Silling and R. Lehoucq. "Convergence of peridynamics to classical elasticity theory". In: Journal of Elasticity 93.1 (2008), pp. 13-37. ISSN: 0374-3535. DOI: $10.1007 /$ s10659-008-9163-3. URL: http://link. springer.com/10.1007/s10659-008-9163-3. 
[12] Erdogan Madenci and Erkan Oterkus. Peridynamic Theory and Its Applications. Vol. 1. Springer, New York, 2014. ISBN: 978-1-4614-8464-6. DOI: 10.1007/978-1-4614-8465-3. eprint: arXiv:1011.1669v3.

[13] Stewart A. Silling and E. Askari. "A meshfree method based on the peridynamic model of solid mechanics". In: Computers and Structures 83.17-18 (2005), pp. 1526-1535. ISSN: 00457949. DOI: $10.1016 / \mathrm{j}$. compstruc.2004.11.026.

[14] M. Ghajari, L. Iannucci, and P. Curtis. "A peridynamic material model for the analysis of dynamic crack propagation in orthotropic media". In: Computer Methods in Applied Mechanics and Engineering 276 (2014), pp. 431-452. ISSN: 0045-7825. DOI: $10.1016 / \mathrm{j}$. cma.2014.04.002. URL: http://dx.doi.org/10.1016/j.cma.2014.04.002.

[15] Daniele Dipasquale et al. "A discussion on failure criteria for ordinary state-based Peridynamics". In: Engineering Fracture Mechanics 186 (2017), pp. 378-398. ISSN: 0013-7944. DOI: 10 .1016/j.engfracmech . 2017 .10.011. URL: http : / www . sciencedirect . com/ science/article/pii/S0013794417303478?via\%3Dihub.

[16] John T. Foster, Stewart A. Silling, and Wayne W. Chen. "State Based Peridynamic Modeling of Dynamic Fracture". In: Proceedings of the SEM Annual Conference, Albuquerque, USA. 2009, pp. 1-6.

[17] Martin Rädel and Christian Willberg. PeriDoX. GitHub repository. Mar. 2018. DOI: 10. 5281/zenodo.1403015. URL: https://github.com/PeriDoX/PeriDoX.

[18] Adair R. Aguiar and Roger Fosdick. "A constitutive model for a linearly elastic peridynamic body". In: Mathematics and Mechanics of Solids 19.5 (2014), pp. 502-523. ISSN: 1081-2865. DOI: $10.1177 / 1081286512472092$.

[19] T. L. Anderson. Fracture Mechanics: Fundamentals and Applications, Third Edition. CRC Press, 2005. ISBN: 9781420058215.

[20] S. H. Song. "Fracture of asphalt concrete: a cohesive zone modeling approach considering viscoelastic effects". PhD thesis. University of Illinois at Urbana-Champaign, 2006.

[21] Michael L. Parks et al. Peridigm Users Guide v1.0.0. Tech. Report SAND2012-7800. Sandia report. Albuquerque, New Mexico 87185 and Livermore, California 94550, USA, 2012. URL: http://www.sandia.gov/ djlittl/docs/PeridigmV1.0.0.pdf.

[22] S. G. Lekhnitskii. Anisotropic Plates. Gordon and Breach Science Publishers, 1968. ISBN: 9780677206707.

[23] J.P.R. Monteiro et al. "Experimental estimation of the mechanical and fracture properties of a new epoxy adhesive". In: Applied Adhesion Science 3.1 (2015), p. 25. ISSN: 2196-4351. DOI: 10 .1186/s40563-015-0056-y. URL: https : //doi .org/10 .1186/s40563-0150056-y.

[24] J.M. Melenk and I. Babuška. "The partition of unity finite element method: Basic theory and applications". In: Computer Methods in Applied Mechanics and Engineering 139.1 (1996), pp. 289-314. ISSN: 0045-7825. DOI: https://doi.org/10.1016/S0045-7825(96) 01087-0. URL: http: //www. sciencedirect.com/science/article/pii/S0045782596010870.

[25] Ted Belytschko, Robert Gracie, and Giulio Ventura. "A Review of Extended/Generalized Finite Element Methods for Material Modelling". In: Modelling and Simulation in Materials Science and Engineering 17.4 (2009), p. 043001. ISSN: 1361-651X. DOI: 10.1088/09650393/17/4/043001.

[26] Christian Willberg and Martin Rädel. "An energy based peridynamic state-based failure criterion". In: PAMM-Proceedings, Applied Mathematics and Mechanics (submitted). 2018.

[27] A. Trilaksono et al. "Continuous Damage Monitoring of a Thin Composite Structural with Mismatched Stiffener in a Combined Joint Using Fiber Bragg Grating under Tension and 
Three-Point Loading". In: Open Journal of Composite Materials 3.3 (2013). DoI: 10.4236/ oj cm.2013.33008.

[28] Daniel Krause. "A physically based micromechanical approach to model damage initiation and evolution of fiber reinforced polymers under fatigue loading conditions". In: Composites Part B: Engineering 87.Supplement C (2016), pp. 176-195. ISSN: 1359-8368. DOI: 10 . 1016/j . compositesb.2015.10.012. URL: http://www . sciencedirect.com/science/ article/pii/S1359836815006307.

[29] M. Rädel, C. Willberg, and J. Schmidt. "Effect of Discretization and Stochastic Material Distribution on Crack Initiation in Peridynamics". In: 14th U.S. National Congress on Computational Mechanics 2017. 2017.

[30] M. Rädel et al. "Influence of propabilistic material distribution in peridyamics to the crack initiation". In: 6th ECCOMAS Thematic Conference on the Mechanical Response of Composites COMPOSITES 2017. 2017.

German Aerospace Center,, Institute of Composite Structures and Adaptive Systems, Lilienthalplatz 7, Braunschweig, 38108, Germany

Email address: christian.willberg@dlr.de

German Aerospace Center, Institute of Composite Structures and Adaptive Systems, LilienTHAlplatz 7, Braunschweig, 38108, Germany

Email address: Lasse@wiedemann-sh.de

German Aerospace Center, Institute of Composite Structures and Adaptive Systems, LilienThalplatz 7, Braunschweig, 38108, Germany

Email address: martin.raedel@dlr.de 